
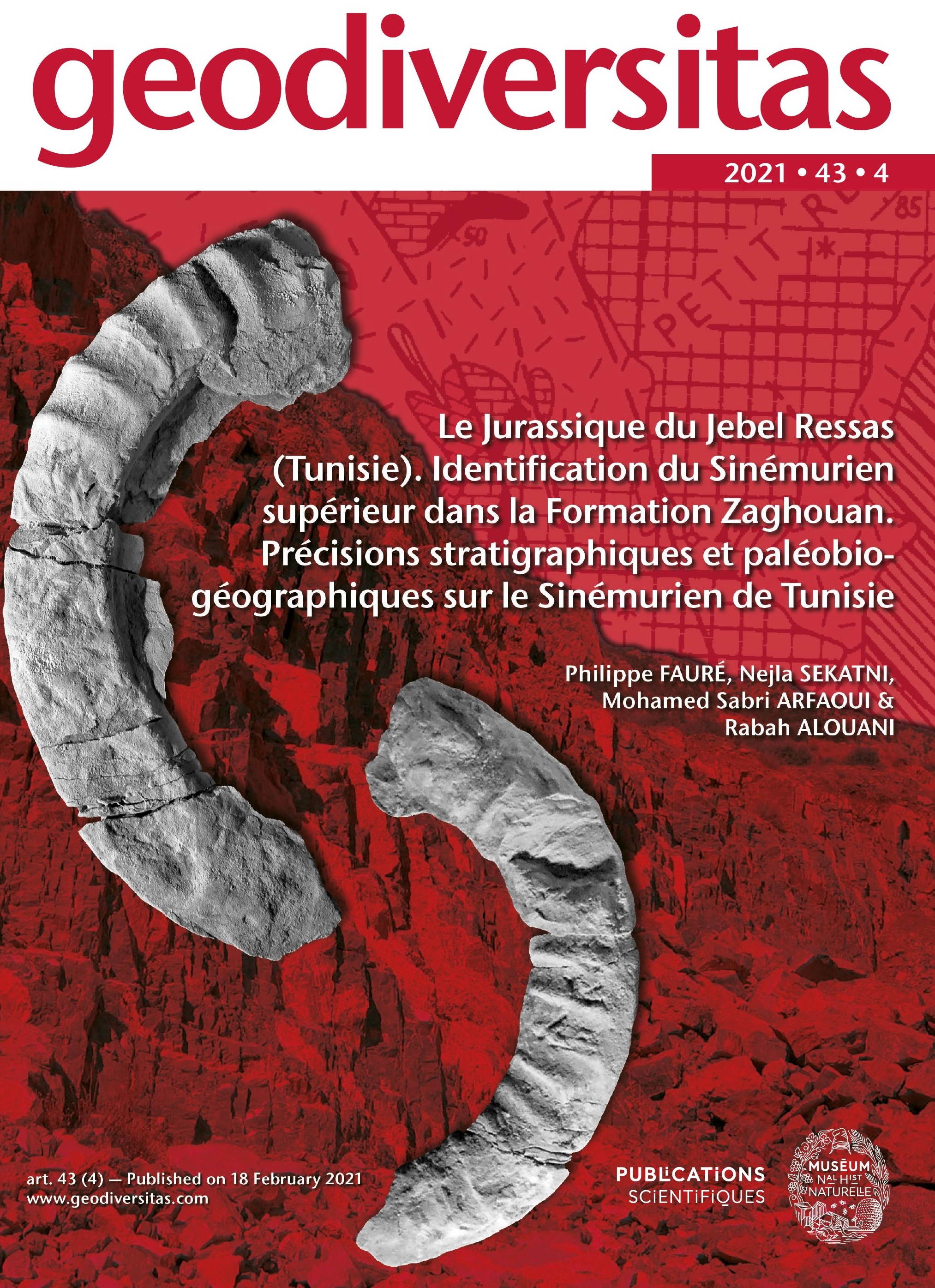
DiRECTEUR DE LA PUBLICATION / PUBLICATION DIRECTOR: Bruno David, Président du Muséum national d'Histoire naturelle

RÉdACTEUR EN CHEF / EDITOR-IN-CHIEF: Didier Merle

ASSISTANT DE RÉDACTION / ASSISTANT EDITOR: Emmanuel Côtez (geodiv@mnhn.fr)

Mise EN PAGE / PAGE LAYOUT: Emmanuel Côtez

COMITÉ SCIENTIFIQUE / SCIENTIFIC BOARD:

Christine Argot (Muséum national d'Histoire naturelle, Paris)

Beatrix Azanza (Museo Nacional de Ciencias Naturales, Madrid)

Raymond L. Bernor (Howard University, Washington DC)

Alain Blieck (chercheur CNRS retraité, Haubourdin)

Henning Blom (Uppsala University)

Jean Broutin (Sorbonne Université, Paris, retraité)

Gaël Clément (Muséum national d'Histoire naturelle, Paris)

Ted Daeschler (Academy of Natural Sciences, Philadelphie)

Bruno David (Muséum national d'Histoire naturelle, Paris)

Gregory D. Edgecombe (The Natural History Museum, Londres)

Ursula Göhlich (Natural History Museum Vienna)

Jin Meng (American Museum of Natural History, New York)

Brigitte Meyer-Berthaud (CIRAD, Montpellier)

Zhu Min (Chinese Academy of Sciences, Pékin)

Isabelle Rouget (Muséum national d'Histoire naturelle, Paris)

Sevket Sen (Muséum national d'Histoire naturelle, Paris, retraité)

Stanislav Štamberg (Museum of Eastern Bohemia, Hradec Králové)

Paul Taylor (The Natural History Museum, Londres, retraité)

COUVERTURE / COVER:

Réalisée à partir des Figures de l'article/Made from the Figures of the article.

Geodiversitas est indexé dans / Geodiversitas is indexed in:

- Science Citation Index Expanded (SciSearch ${ }^{\circledR}$ )

- ISI Alerting Services ${ }^{\circledR}$

- Current Contents ${ }^{\circledR}$ / Physical, Chemical, and Earth Sciences ${ }^{\circledR}$

- Scopus ${ }^{\circledR}$

Geodiversitas est distribué en version électronique par / Geodiversitas is distributed electronically by:

- BioOne ${ }^{\circledR}$ (http://www.bioone.org)

Les articles ainsi que les nouveautés nomenclaturales publiés dans Geodiversitas sont référencés par / Articles and nomenclatural novelties published in Geodiversitas are referenced by:

- ZooBank ${ }^{\circledR}$ (http://zoobank.org)

Geodiversitas est une revue en flux continu publiée par les Publications scientifiques du Muséum, Paris Geodiversitas is a fast track journal published by the Museum Science Press, Paris

Les Publications scientifiques du Muséum publient aussi / The Museum Science Press also publish: Adansonia, Zoosystema, Anthropozoologica, European Journal of Taxonomy, Naturae, Cryptogamie sous-sections Algologie, Bryologie, Mycologie, Comptes Rendus Palevol

Diffusion - Publications scientifiques Muséum national d'Histoire naturelle

CP $41-57$ rue Cuvier F-75231 Paris cedex 05 (France)

Tél. : 33 (0)1 40794805 / Fax: 33 (0)14079 3840

diff.pub@mnhn.fr / http://sciencepress.mnhn.fr

(C) Publications scientifiques du Muséum national d'Histoire naturelle, Paris, 2021

ISSN (imprimé / print): 1280-9659/ ISSN (électronique / electronic): 1638-9395 


\title{
Le Jurassique du Jebel Ressas (Tunisie). Identification du Sinémurien supérieur dans la Formation Zaghouan. Précisions stratigraphiques et paléobiogéographiques sur le Sinémurien de Tunisie
}

\author{
Philippe FAURÉ \\ Centre d'ACP, 47 rue Théron Périé, BP 30205 , \\ F-81104 Castres cedex (France) \\ et Muséum d'Histoire Naturelle de Toulouse, \\ F-31000, Toulouse (France) \\ philipfaure@wanadoo.fr \\ Nejla SEKATNI \\ Université de Sousse, faculté des Lettres et des Sciences \\ Humaines de Sousse, Département de géographie \\ Cité Erriadh, 4029 Sousse Ryadh, Sousse (Tunisie) \\ sgatninejla@yahoo.fr \\ Mohamed Sabri ARFAOUI \\ Département de géologie, faculté des sciences de Tunis, \\ 1060 El Manar II, Tunis (Tunisie) \\ sabriarfaoui@yahoo.fr \\ Rabah ALOUANI \\ Département de géologie, faculté des sciences de Bizerte, \\ Université de Carthage \\ 7021 Zerzouna Bizerte (Tunisie) \\ rabeh_alouani@yahoo.fr
}

MOTS CLÉS

Téthys,

Jebel Ressas,

Jurassique inférieur,

Sinémurien,

ammonites,

biostratigraphie, paléobiogéographie.
urn:Isid:zoobank.org:pub:D3197095-E2DE-44AD-AA1D-80A8781199C0

Fauré Ph., Sekatni N., Arfaoui M. S. \& Alouani R. 2021. - Le Jurassique du Jebel Ressas (Tunisie). Identification du Sinémurien supérieur dans la Formation Zaghouan. Précisions stratigraphiques et paléobiogéographiques sur le Sinémurien de Tunisie. Geodiversitas 43 (3): 95-119. https://doi.org/10.5252/geodiversitas2021v43a4. http://geodiversitas.com $/ 43 / 4$

RÉSUMÉ

La révision de la succession lithostratigraphique du Jurassique du Jebel Ressas (Dorsale tunisienne, Tunisie) permet d'identifier toutes les formations déjà définies par ailleurs dans la Dorsale tunisienne. La Formation Zaghouan y est nouvellement datée du Sinémurien supérieur par des ammonites appartenant aux chronozones à Obtusum (sous-chronozone à Stellare) et à Raricostatum (sous-chronozone à Raricostatum). Six taxons d'Ammonitina appartenant aux genres Asteroceras (A. gr. saltriense (Parona, 1896), A. cf. meridionalis Dommergues, Meister \& Mettraux, 1990), Arnioceras (A. gr. ceratitoides (Quenstedt, 1849) - rejectum Fucini, 1902), Epophioceras (E. cf. landrioti (d'Orbigny, 1850)) et Paltechioceras (P. bavaricum (Böse, 1894), P. cf. charpentieri (Schafhäult, 1847)) sont décrits et figurés. Ces ammonites permettent de corréler la «Formation Zaghouan» avec les «Niveaux condensés du sommet de la Formation Oust», déjà bien connus pour leur riche ammonitofaune sinémurienne et dont les âges sont ici réévalués. Les affinités paléobiogéographiques des ammonites du Sinémurien de Tunisie sont discutées. 


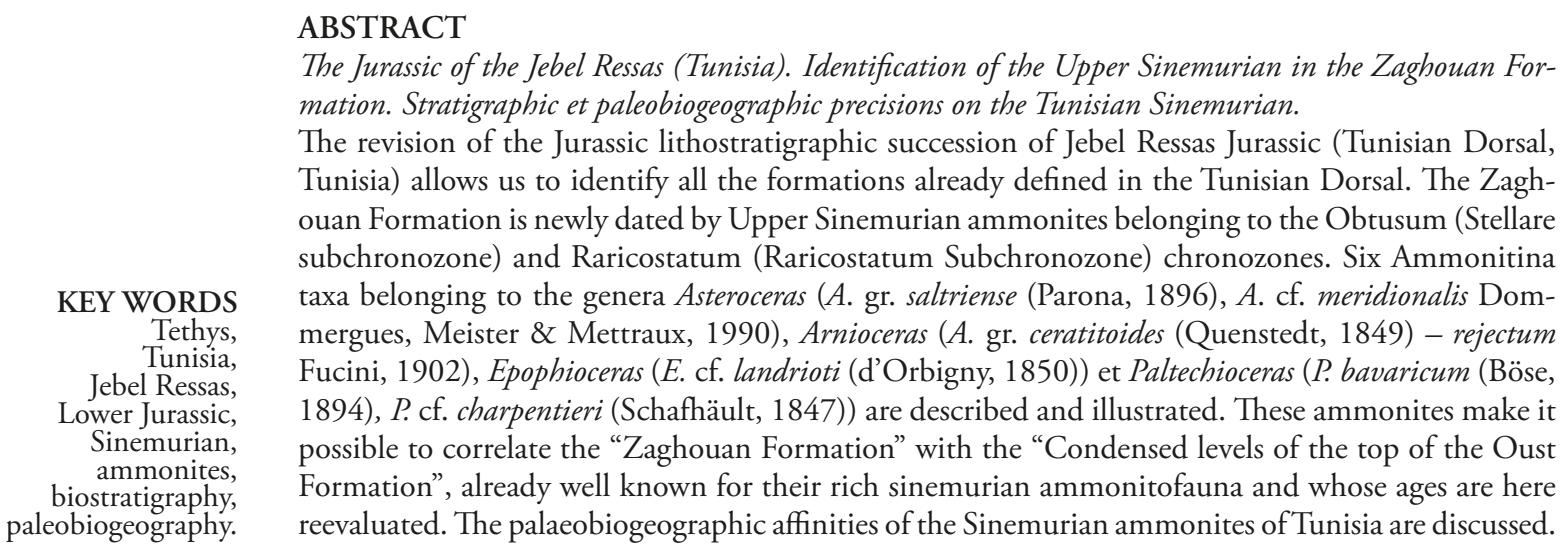

\section{INTRODUCTION}

La Dorsale tunisienne est l'élément orographique et structural le plus marquant de la Tunisie atlasique. Une succession de reliefs, souvent très accusés, s'y alignent selon une direction SW-NE, le long de la grande faille de Zaghouan (Fig. 1). Le Jebel Ressas en est l'un des plus spectaculaires reliefs. Situé au nord de la Dorsale, à $15 \mathrm{~km}$ au sud du Golfe de Tunis, sa pyramide déchiquetée caractéristique s'élève brusquement au signal du Grand Ressas, jusqu'à près de $800 \mathrm{~m}$ d'altitude au-dessus de la plaine de Mornag.

Les travaux de révision de la stratigraphie des jebels les plus septentrionaux de la Dorsale (Fauré et al. 2007; Sekatni et al. 2008 ; Sekatni-Aich 2010; Sekatni et al. 2013) ont été pour nous l'occasion d'une révision de la stratigraphie du Jurassique du Jebel Ressas dont aucune coupe continue n'avait été publiée et dont la succession stratigraphique restait confuse et peu précise. Nous l'exposons dans une première partie de ce travail.

La découverte, dans les calcaires à silex de la Formation (Fm.) Zaghouan du Jebel Ressas, de plusieurs associations d'ammonites du Sinémurien supérieur retiendra ensuite toute notre attention car cette formation, qui intéresse la seule partie septentrionale de la Dorsale tunisienne, demeurait à ce jour non ou très partiellement datée. Son équivalence latérale avec les "Niveaux condensés du sommet de la Formation Oust», célèbres pour leurs riches faunes d'ammonites du Sinémurien supérieur et du Pliensbachien inférieur dans les jebels proches, Oust et Zaghouan, avait été déjà suggérée par les auteurs (Rakús \& Biely 1970; Fauré \& Peybernès 1986a, b; Rakús \& Guex 2002), mais n'avait à ce jour pu être confirmée. Ces faits nouveaux nous autorisent ainsi de nouvelles corrélations au sein du Jurassique tunisien. Nous synthétiserons ensuite toutes les données sur la stratigraphie du Sinémurien tunisien et discuterons de ses affinités paléobiogéographiques.

\section{LE JEBEL RESSAS. ÉTUDE LITHOSTRATIGRAPHIQUE DU JURASSIQUE}

Le Jebel Ressas est une masse carbonatée étroite, allongée selon un axe SSW-NNE, de $3 \mathrm{~km}$ de longueur et $1 \mathrm{~km}$ de largeur, environ, constituée de calcaires du Jurassique, subverticaux à légèrement déversés vers le sud-ouest. Le Jurassique inférieur constitue l'ossature du Grand Ressas, tandis que le Petit Ressas est composé de calcaires récifaux du Jurassique supérieur (Fig. 2).

\section{HISTORIQUE DES RECHERCHES}

Par sa proximité de la ville de Tunis et surtout en raison de ses riches gisements plombo-zincifères, le Jebel Ressas a, depuis le 19 ème siècle, attiré de nombreux chercheurs, essentiellement des géologues miniers. Le Jurassique supérieur y est identifié dès 1884 par Meneghini, mais c'est Pervinquière (1903) qui montre que le massif est essentiellement constitué de calcaires du Jurassique. Dans ces calcaires, il démontre la présence du Lias et du Tithonique. Solignac (1927) en donne l'étude stratigraphique et structurale la plus complète et affirme que "la masse de cette montagne est surtout liasique». Il y identifiera le Toarcien, l'Aalénien, ainsi que l'«Argovien rouge" (Oxfordien) dans le complément de son ouvrage sur la tectonique du massif. Le Bajocien sera mis en évidence plus tard par Castany (1955). Grace à la micropaléontologie, Bonnefous (1972) suggère l'âge Hettangien à Sinémurien de la masse des formations calcaréo-dolomitiques qui constituent l'ossature du Ressas. Avec l'établissement de la carte géologique de Grombalia au 1/50.000ème, on peut considérer que le cadre structural et la stratigraphie du Jurassique du Ressas sont totalement aboutis (Bujalka et al. 1971; Rakús 1973) (Fig. 2). Rakús \& Guex (2002) vont ensuite souligner les affinités lithologiques entre la 


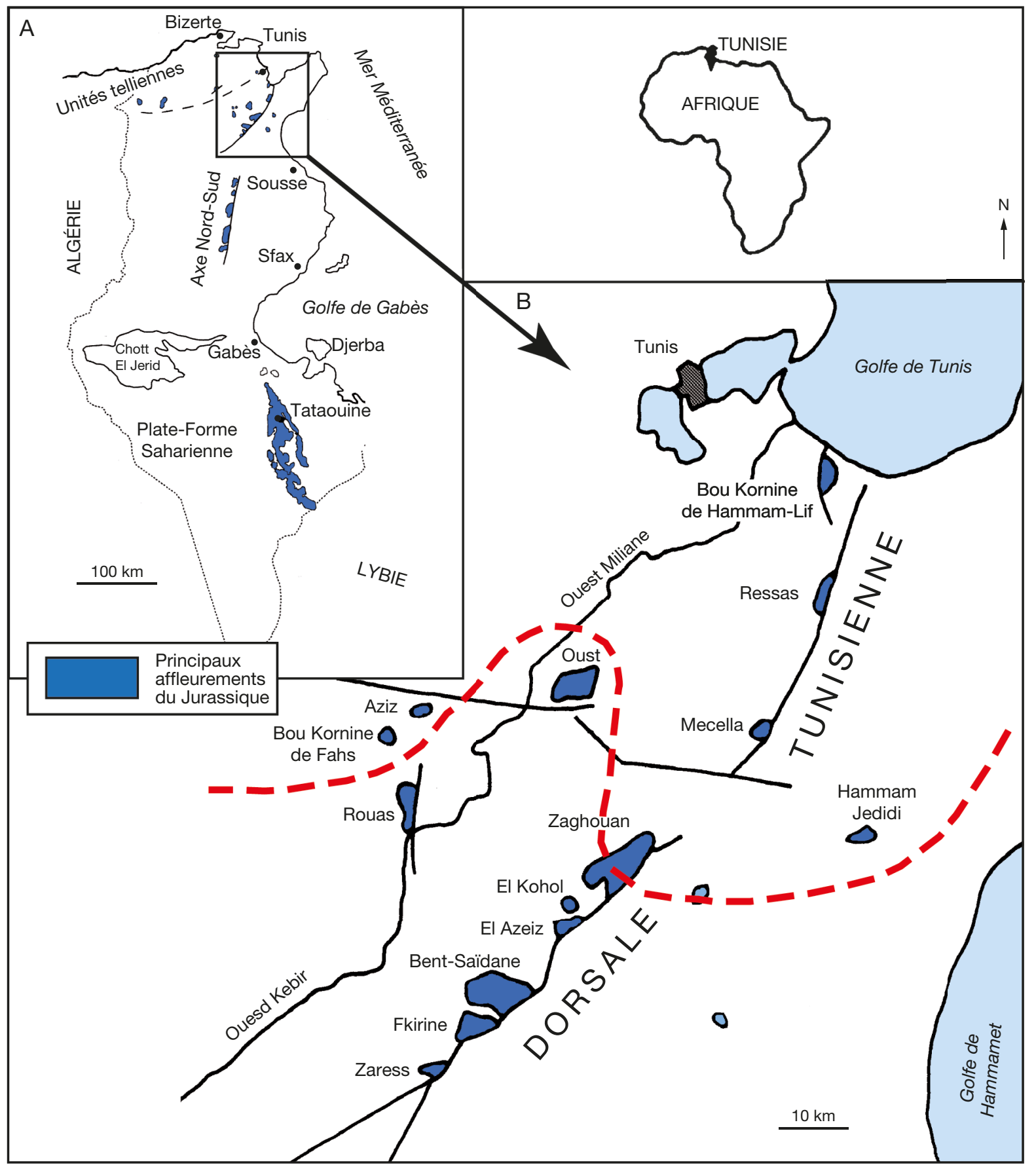

FIG. 1. - A, carte géologique synthétique de la Tunisie septentrionale et localisation des principaux affleurements de Jurassique; B, carte structurale schématique de la partie nord-est de la Tunisie. Trait en pointillés rouges: limite entre les faciès pélagiques de calcaires à silex de la Fm Zaghouan, au nord, et les faciès condensés des Niveaux condensés du sommet de la Formation Oust, au sud.

succession du Ressas et celle des jebels voisins, en particulier celle du proche Jebel Zaghouan et vont préciser certains points de la stratigraphie du Pliensbachien et du Bajocien du massif, que nous évoquerons plus loin. Ils y identifient nouvellement le Bathonien inférieur.

\section{ÉTUDE STRATIGRAPHIQUE}

Cette étude est permise par les exceptionnelles conditions d'observation offertes par le niveau supérieur d'exploitation de la grande carrière du Ressas (septembre 2005), qui entaille le versant oriental du jebel, à son extrémité sud-occidentale, au lieu-dit «Paroi Rouge» (Fig. 3A). Les bancs y affleurent avec un pendage vertical, offrant de remarquables conditions d'affleurement (Fig. 3B). Cette coupe nous permet l'identification et la datation de la plupart des unités lithostratigraphiques reconnues dans la Dorsale tunisienne (Fauré \& Peybernès 1986a, b; Rakús \& Guex 2002; Soussi 2002; Fauré et al. 2007; Sekatni et al. 2008, 2013) (Fig. 4). 


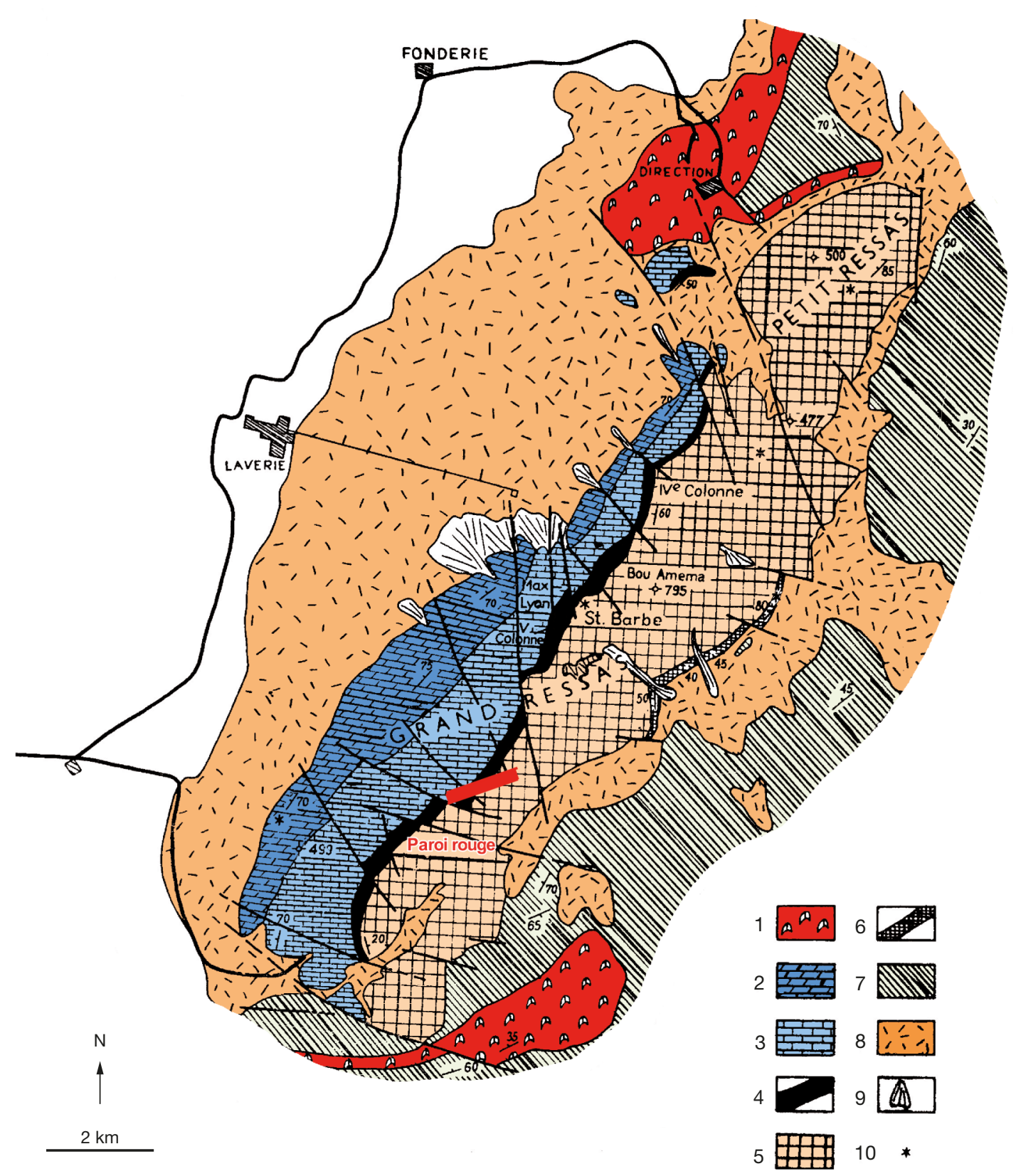

FIG. 2. - Carte géologique du Jebel Ressas, reproduite de Rakús (1973: fig. 1). Légende: 1, argiles bariolées du Trias supérieur; 2, série dolomitique inférieure (Fm Oust pars); 3, série carbonatée supérieure (Fm Oust pars); 4, calcaires à silex (Fm Zaghouan), calcaires marneux (Fm Bou Gabrine), marnes (Fm Stah), calcaires à zoophycos (Fm Kef el Orma) et calcaires noduleux pseudoconglomératiques rouges (Fm Zaress); 5, Calcaires du Ressas (Tithonique); 6, calcaires foncés (Berriasien); 7, marnes et calcaires marneux (Valanginien); 8 , éboulis; 9 , déblais d'exploitations; 10, gites fossilifères.

La Formation Oust (Rakús \& Biely 1970) (287 m):

Nous ne préciserons pas ici la stratigraphie de cette puissante formation carbonatée qui constitue l'ossature du jebel. Elle est bien décrite par Bismuth et al. (1967), puis Bonnefous (1972) et Rakús (1973), ouvrages auxquels nous renvoyons le lecteur. Rappelons que les auteurs s'accordent pour reconnaître deux séries principales:

- Une série dolomitique inférieure ( $80 \mathrm{~m})$, faite de brèches dolomitiques et de dolomies grises à rousses, compactes ou litées à fantômes d'algues (Collenia, Thaumatoporella) et foraminifères Verneuilinidae.

- Une série carbonatée supérieure (207 m), très massive, constituée de calcaires organo-détritiques gris à algues
[Thaumatoporella cf. parvovesiculifera (Reineri, 1922); Palaeodasycladus cf. mediterraneus Pia, 1920 ; Cayeuxia liassica Le Maître, 1935]. Le sommet de la formation montre, sur $10 \mathrm{~m}$ d'épaisseur, la superposition de bancs de calcaire oobioclastique, de calcaire dolomitique à lamines algaires planes-parallèles ou ondulées et de dolomie laminée à niveaux de "bird eyes", s'enchaînant en séquences régressives superposées dans un environnement de plate-forme interne margino-littorale.

La Formation, dont l'âge Trias supérieur (Rhétien) à Sinémurien inférieur est admis (Soussi 2002), est surmontée par une surface ondulée, karstifiée, témoignant d'un arrêt prolongé de la sédimentation. 

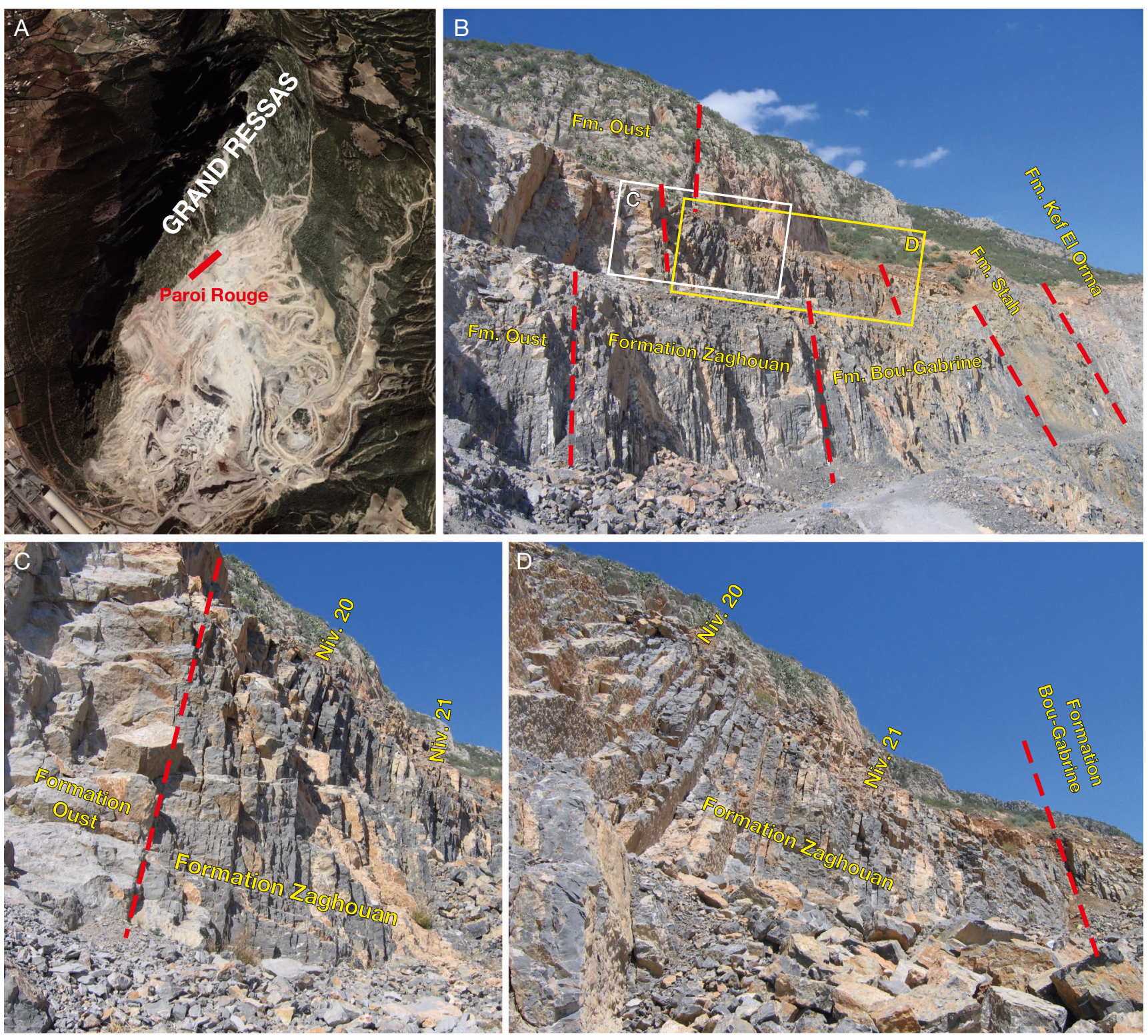

FIG. 3. - A, photographie aérienne du Grand Ressas (d'après Google Earth). Localisation de la coupe étudiée au lieu-dit Paroi rouge, au niveau du gradin le plus élevé de la carrière du Ressas; B, vue générale de la coupe de Paroi Rouge: Trait pointillés rouges, limites des formations lithostratigraphiques. Les encadrés $C$ et $\mathrm{D}$ matérialisent les gros plans suivants: C, photographie du sommet de la Fm Oust et de la partie inférieure de la Fm Zaghouan; $\mathbf{D}$; photographie de la partie supérieure de la Fm Zaghouan et de la base de la Fm Bou Gabrine.

La Formation Zaghouan (Fauré \& Peybernès 1986a) (15 m) (Figs 3, 4)

Calcaires bruns à silex noirs, disposés en bancs réguliers, de 20 à $50 \mathrm{~cm}$ d'épaisseur, à surfaces ondulées ferrugineuses (Fig. 3). La faune y est rare, quelques bélemnites, bivalves et de rares ammonites phosphatées, mal conservées, aplaties et déformées, toujours situées à la surface des bancs, témoignant entre chacun, d'un épisode de condensation sédimentaire. On relève (Fig. 4):

- niv. 20: Asteroceras gr. saltriensis Parona, 1896, A. cf. meridionalis Dommergues, Meister \& Mettreaux, 1990, Epophioceras aff. landrioti (d'Orbigny, 1850) et Arnioceras gr. ceratitoides (Quenstedt, 1849) - rejectum Fucini, 1902, association que nous plaçons dans la partie supérieure de la sous-chronozone à Stellare (chronozone à Obtusum, Sinémurien supérieur).

- niv. 21: Paltechioceras bavaricum (Böse, 1894), P. cf. charpentieri (Schafhäult, 1847) et Juraphyllites aff. nardii (Meneghini, 1853), association de la chronozone à Raricostatum, vraisemblablement légèrement condensée, que nous plaçons à l'articulation de la sous-chronozone à Raricostatum et de la sous-chronozone à Macdonnelli (Sinémurien supérieur).

La Formation est surmontée par une discontinuité plane nette. Soulignons que les calcaires à silex, que nous plaçons ici dans la Fm Zaghouan, avaient été reconnus mais non datés par Rakús (1973). 
La Formation Bou Gabrine (Fauré et al. 2007) (31 m) (Figs 3, 4) Trois ensembles lithologiques, séparés par des discontinuités mineures se succèdent, de bas en haut:

$-13 \mathrm{~m}$ : Ensemble induré de calcaires argileux gris, disposés en bancs compacts ou noduleux, bioturbés, à surface ondulée, parfois séparés par de fins lits marneux.

Malgré l'absence de faune, nous proposons pour ce premier ensemble, un âge Pliensbachien inférieur terminal (chronozone à Davoei = zone à Dilectum sensu Braga et al. 1982) à début de Pliensbachien supérieur (partie inférieure de chronozone à Margaritatus, sous-chronozone à Stokesi = zone à Lavinianum sensu Braga et al. 1982), par corrélation avec des couches homologues du Jebel Zaghouan (est du Poste optique) (Fauré et al. 2007).

$-12,5 \mathrm{~m}$ : Calcaires argileux gris, noduleux, tendres disposés en bancs noduleux de 10 à $15 \mathrm{~cm}$ d'épaisseur, séparés par des lits stratocroissants de marne grise ou jaune. Dans la partie médiane de cette assise (niv. 30), les ammonites Leptaleoceras accuratum (Fucini, 1931) et Arieticeras gr. algovianum (Oppel, 1862) signalent la partie supérieure de la chronozone à Margaritatus (zone à Algovianum, sensu Braga et al. 1982), du Pliensbachien supérieur. Rappelons que l'ammonite indice avait été déjà signalée par Rakús (1973), à «Paroi Rouge», figurée par Rakús \& Guex (2002: pl. 27, fig. 8) et interprétée comme Amaltheus margaritatus de Montfort, 1808 forme idrissensis Faugères, 1978 par Fauré et al. (2007).

$-6 \mathrm{~m}$ : Bancs compacts, plus en relief, de calcaire argileux séparés par des joints argileux. L'ammonite Argutarpites decoratum (Fucini, 1924) de la partie supérieure de la chronozone à Margaritatus, y est récoltée niv. 35 (Fauré et al. 2007: fig. 6J). La chronozone à Spinatum (= zone à Emaciatum sensu Braga et al. 1982) est identifiée dans des couches homologues du Jebel Zaghouan (est du Poste Optique) (Fauré et al. 2007).

La Formation est surmontée par une discontinuité sédimentaire bien marquée à partir de laquelle le régime sédimentaire devient essentiellement argilo-carbonaté.

\section{La Formation Stah (Fauré \& Peybernès 1986a sensu Rakuis \& Guex 2002) (18 m)}

Cette assise est rarement visible car le plus souvent, à la fois oblitérée par la tectonique et recouverte par les éboulis. Elle offre, dans la carrière de Paroi Rouge, des conditions d'affleurement exceptionnellement bonnes. Il s'agit d'une alternance rythmique régulière de bancs de calcaire argileux plus ou moins fortement noduleux et de lits de marne beige, silteuse. Les ammonites Hildoceras lusitanicum Meister, 1913 et Harpoceras mediterraneum Pinna, 1968, du Toarcien moyen, chronozone à Bifrons, sont récoltées, hors place (niv. 40) dans le tiers inférieur de la formation.

Au 13ème mètre à partir de sa base, $2 \mathrm{~m}$ de calcaires particulièrement noduleux, voire pseudo-conglomératiques, légèrement ferrugineux, approchent le faciès "ammonitico rosso». Ces bancs n'ont pas fourni d'ammonite.
La Formation Kef el Orma (Fauré \& Peybernès 1986a) (20 m) La sédimentation devient ensuite progressivement carbonatée et montre, de bas en haut:

$-2,5 \mathrm{~m}$ : Calcaires argileux noduleux clairs, très bioturbés, en bancs irréguliers, surmontés par des surfaces ferrugineuses ondulées.

- 11,5 m: Calcaires argileux bioturbés, disposés en bancs ondulés ou à débit noduleux, d'épaisseur irrégulière. Cette assise est intercalée de trois niveaux conglomératiques situés à $0,50 \mathrm{~m}, 3 \mathrm{~m}$ et $7,5 \mathrm{~m}$ de la base de l'assise. Tous sont, à l'identique, constitués de galets carbonatés polygéniques non cohésifs, noyés dans une matrice carbonatée ou argilo-carbonatée bien stratifiée. Leur faciès est identique à celui des niveaux conglomératiques déjà décrits, dans cette formation, dans le Jebel Bou Kornine de Hammam-Lif où ils s'échelonnent du Toarcien supérieur, chronozone à Aalensis, à l'Aalénien supérieur, chronozone à Comptum (Sekatni et al. 2008).

- $6 \mathrm{~m}$ : Calcaires biodétritiques à Zoophycos en bancs épais et compacts. L'ammonite Emileites sp. signale la présence dans ces bancs du Bajocien inférieur, chronozone à Laeviuscula, sous-chronozone à Ovalis.

De la formation proviennent très vraisemblablement " Tmetoceras sp. cf. T. regleyi (Thiollière)" et "Leioceras sp. ?» de l'Aalénien, respectivement signalés par Solignac (1927) et Rakús (1973).

L'âge Toarcien supérieur à Bajocien inférieur de la formation, est le même que dans les autres jebels de la Dorsale (Sekatni et al., 2008; Sekatni-Aich 2010). Elle est tronquée à son sommet par une discontinuité plane correspondant à un hard-ground.

\section{La Formation Bent Saïdane (Peybernès 1992)}

Elle est absente à Paroi Rouge, oblitérée par le contact apparemment stratigraphique de l'«ammonitico rosso » oxfordien sur les calcaires bajociens inférieurs. L'intervalle Bajocien supérieur-Callovien est ainsi totalement absent.

Des ammonites appartenant à cette tranche d'âge sont cependant signalées par Rakús (1973) et Rakús \& Guex (2002) à proximité des travaux miniers Max-Lyon, avec: Kumatostephanus (Stematoceras) cf. frechi (Renz, 1913) et Holcophylloceras cf. mediterraneum (Neumayr, 1871) du Bajocien moyen, chronozone à Humphriesianum; Stephanoceras sp., Dimorphinites cf. dimorphus (d'Orbigny, 1846), Cadomites sp. gr. daubegnyi (Gemmellaro, 1877) du Bajocien supérieur, chronozone à Parkinsoni; Morphoceras cf. macrescens (Buckman, 1923) du Bathonien inférieur, chronozone à Zigzag. Ces ammonites proviennent d'un sédiment "pseudobréchoïde» dont Rakús (1973) constatait déjà l'analogie avec les «pseudobrèches du Djebel Bou Kornine d'Hammam-Lif». Ce faciès noduleux et pseudo-conglomératique est de toute évidence identique à celui des "Conglomérats supérieurs» de ce Jebel dont nous avons montré qu'il s'agissait d'un faciès tectogène de la Fm Bent Saïdane (Sekatni et al. 2013: 187). Des témoins de cette formation seraient ainsi bien préservés dans la seule partie nord du Jebel.

FIG. 4. - Log stratigraphique du Jurassique (Sinémurien supérieur à Kimméridgien inférieur) du Jebel Ressas au niveau de la coupe de Paroi Rouge. Lithostratigraphie, répartition des ammonites et biozonation standard à l'échelon de la chronozone d'ammonites. En gras, chronozones identifiées dans le Jebel Ressas; en italique, chronozones déduites de la comparaison avec les Jebels Bou Kornine de Hammam-Lif et Zaghouan. Échelle: 3 m. 


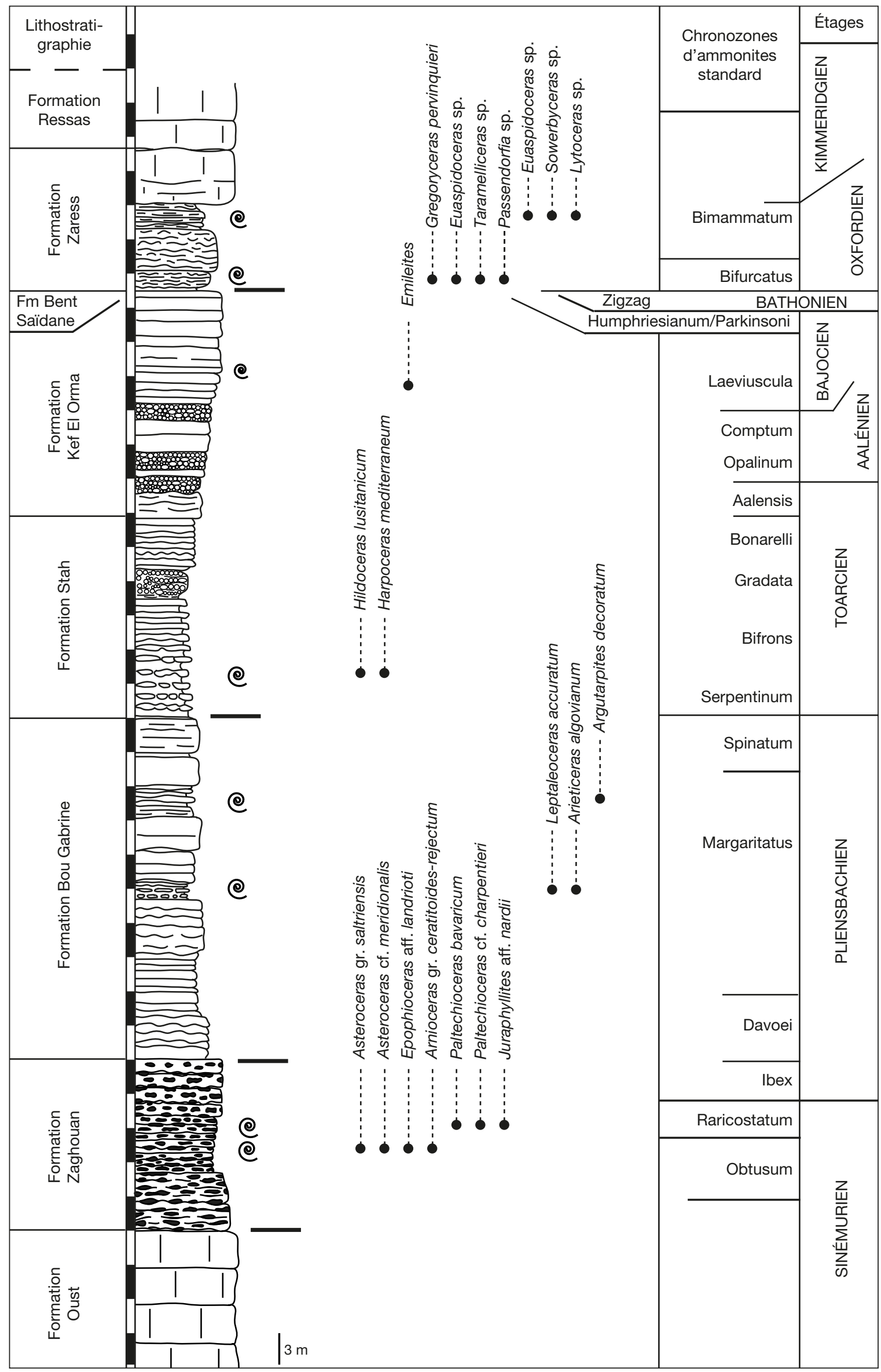


La Formation Zaress (Peybernès 1992) (7 m)

À partir de la discontinuité sédimentaire très marquée qui surmonte les calcaires à Zoophycos de la Formation Kef el Orma, on distingue de bas en haut:

$-1 \mathrm{~m}$ (niv. 50$)$ : calcaires noduleux gris ou verdâtres à passées pseudo-conglomératiques, intercalés de fins niveaux noduleux rouges, très riches en ammonites, donnant à l'ensemble l'aspect d'un "ammonitico rosso " et dans lesquels on récolte Gregoryceras pervinquieri Spath, 1913, Euaspidoceras sp., Taramelliceras sp., Passendorfia (P.) sp., Oppelidés s.l., Perisphinctidés s.l., ammonites de l'Oxfordien supérieur, chronozone à Bifurcatus, sous-chronozone à Stenocycloides.

$-4,5 \mathrm{~m}$ : Calcaires gris en bancs ondulés ou noduleux de 10 à $20 \mathrm{~cm}$ d'épaisseur, sans fossile.

- 1,5 m (niv. 53): Calcaire noduleux à pseudo-conglomératique, glauconieux à Euaspidoceras sp., Taramelliceras sp., ? Phylloceratidés, ? Sowerbyceras sp., Lytoceras sp., ammonites d'âge Oxfordien supérieur, chronozone à Bimammatum, voire Kimméridgien inférieur.

La limite supérieure de la Formation coïncide avec ce dernier banc de calcaire noduleux. Sans discontinuité apparente, il est suivi par la puissante série des "Calcaires du Ressas", calcaires récifaux en gros bancs compacts (450 m d'épaisseur environ), qui sortent du cadre de notre étude, et dont l'âge Kimméridgien supérieur à Tithonien inférieur des ammonites récoltées de longue date (Pervinquière 1903) est maintenant bien connu (Rakús 1973).

Les ammonites de la Fm Zaress du Djebel Ressas relèvent ainsi du seul Oxfordien supérieur à Kimméridgien inférieur. Rakús (1973) avait déjà constaté le caractère très incomplet de cette formation et avait évoqué sa suppression partielle pour des raisons tectoniques. Nous montrons ici sa parfaite concordance avec la Fm Kef el Orma et la nature stratigraphique du contact entre les deux formations qui correspond à une importante discontinuité sédimentaire qui matérialise la lacune du Bajocien inférieur pars, du Callovien et de l'Oxfordien inférieur.

\section{LE SINÉMURIEN DE TUNISIE}

L'identification nouvelle du Sinémurien supérieur dans la partie moyenne des calcaires à silex de la Fm Zaghouan du Jebel Ressas permet d'envisager la corrélation entre la Formation Zaghouan et les Niveaux condensés du sommet de la Formation Oust, déjà bien connus pour leur riche ammonitofaune sinémurienne (Rakús \& Guex 2002).

Le Lias inférieur est depuis longtemps connu en Tunisie, depuis la découverte par Baltzer (1895) d'ammonites d'affinité sinémurienne sur la coupe "Attaque Angéline», située entre les Jebels Stah et Grand Zaghouan («Arietites conybeari Sowerby, 1816; A. bonardi Sowerby, 1816; A. bucklandi Sowerby, 1816; A. geometricus Oppel, 1856; A. brooki Sowerby, $1818 »)$. Mais le gisement de Baltzer demeure longtemps introuvable et ses déterminations, pourtant effectuées par Mayer-Eymar, ont été mises en doute par Haug (1911: 987) et Castany (1951).
On doit la redécouverte du Sinémurien à Rakús \& Biely (1970), dans les couches condensées qui surmontent les calcaires du Lias inférieur des Jebels Oust et Zaghouan, couches qui étaient jusque-là attribuées au seul Pliensbachien (Dubar 1953). Le Sinémurien tunisien y est exprimé par plusieurs niveaux de condensation très fossilifères dans lesquels ces auteurs identifient alors les "zones à Semicostatum, à Oxynotum et à Raricostatum ". Plusieurs espèces remarquables du Jebel Oust seront ensuite étudiées par Dommergues et al. 1986. C'est surtout à Rakús \& Guex (2002) que l'on doit la description et la figuration exhaustives de cette ammonitofaune riche et très bien conservée, à laquelle on doit la célébrité du Lias de la Dorsale tunisienne.

\section{LE CADRE PALÉOGÉOGRAPHIQUE}

En Tunisie, les dépôts du Sinémurien supérieur sont les premiers sédiments marins francs à ammonites qui expriment, au Lias inférieur, la première submersion marine de la plate-forme continentale qui prolonge l'extrémité occidentale (méditerranéenne) de la Téthys (Fig. 5A). Les ammonites y sont d'emblée nombreuses et diversifiées et les associations se rattachent clairement aux faunes de la Téthys méditerranéenne bien connues au Maghreb (Algérie, Maroc) mais également dans les Apennins, les Cordillères Bétiques... Au sein de cet ensemble, les faunes tunisiennes du Sinémurien supérieur, comme du Pliensbachien inférieur, se distinguent par l'originalité de leurs assemblages et l'abondance des taxons dits endémiques (Rakús \& Guex 2002).

Une importante différenciation paléogéographique des sédiments liasiques résulte d'une première phase d'extension et de la fragmentation de la marge maghrébine de la Téthys occidentale. Dès lors seront distingués en Tunisie (Elmi 2002; Soussi 2002) (Fig. 5B, C) :

- Au sud, une plate-forme saharienne à faciès proximaux et détritiques, appuyée sur le craton africain.

- Au nord, un bassin pélagique subsident, le Sillon tunisien, que l'on peut considérer comme le prolongement oriental d'une branche du sillon atlasique.

- Entre ces deux zones, un «domaine intermédiaire» (Soussi 2002), constitué de plates-formes et de hauts-fonds structurés en blocs basculés.

Au Sinémurien supérieur et au Pliensbachien inférieur, les chaînons jurassiques qui s'égrènent le long de la partie septentrionale de la Dorsale tunisienne se situent à l'articulation entre les deux derniers domaines de sédimentation (Fig. 1, 5C). On distingue:

- Un domaine atlasique méridional, correspondant à la partie centrale de la Dorsale tunisienne, exprimé par les Jebels Oust, Grand Zaghouan, Staa, Azreg, El Kohol, Bent Saïdane et Zaress (Fig. 1), développé en zone de horst, sur une aire de plate-forme soumise à une sédimentation discontinue et condensée.

Les dépôts y sont connus sous le terme de "Niveaux condensés du sommet de la Fm Oust» (Rakús \& Guex 2002). Très fossiliferes, plusieurs niveaux paléontologiques s'y succèdent, échelonnés du Sinémurien (chronozones à Turneri, Obtusum, 


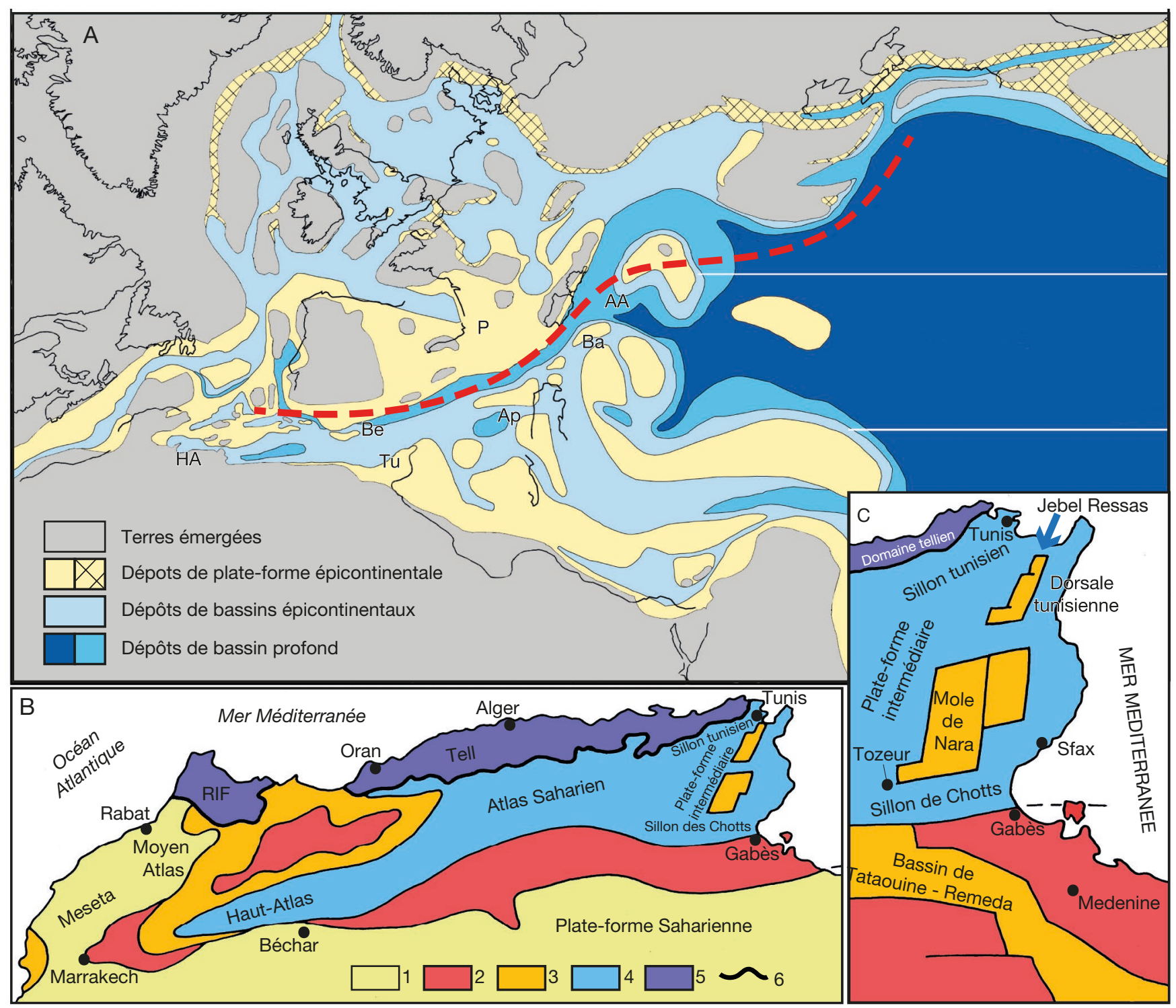

FIG. 5. - A, Cadre paléogéographique simplifié de la Téthys occidentale au Sinémurien (d'après Thierry et al. 2000, modifié). Trait en pointillés rouges: limite approximative entre les biomes téthysien et euro-boréal; B, grandes lignes paléogéographiques du Jurassique d'Afrique du Nord, d'après Elmi (2002); C, schéma paléogéographique de la Tunisie, d'après Soussi (2002). Abréviations: Tu, Tunisie; HA, Haut-Atlas; AA, Austroalpin; B, Monts de Bakony; Py, Pyrénées; Be, Cordillères Bétiques.

Oxynotum et Raricostatum) au Pliensbachien inférieur (chronozones à Jamesoni et à Ibex) (Dubar 1953; Rakús \& Biely 1970 ; Fauré \& Peybernès 1986a, b; Dommergues et al. 1986; Dommergues et al. 2004; Rakús \& Guex 2002).

- Un domaine atlasique septentrional, exprimé dans la partie nord de la Dorsale tunisienne, par les Jebels Ressas, Bou Kornine de Hammam-Lif, Zaghouan (Poste optique), Bou Kornine de Pont de Fahs et Aziz (Rakús \& Biely 1970) (Fig. 1), différencié sur une aire marine profonde, subsidente, à sédimentation pélagique, correspondant au Sillon tunisien.

Les calcaires à lits de silex y sont intégrés dans la Fm Zaghouan (Fauré \& Peybernès 1986a, b). La Formation est aussi présente en Tunisie nord-occidentale, dans les écailles jurassiques qui bordent les grands accidents qui lardent la Tunisie atlasique pré-tellienne (Fauré et al. sous presse).
Âge de la formation Zaghouan en Tunisie ATLAsiQue Les calcaires à silex de la Fm Zaghouan s'avèrent toujours excessivement peu fossilifères et leur datation au «Lotharingien" dans le Jebel Ressas (Rakús \& Biely 1970; Rakús 1973) ne reposait que sur leur seule interposition entre la masse calcaréo-dolomitique du Lias inférieur (Fm Oust) et les calcaires marneux du Pliensbachien (Fm Bou Gabrine). Rappelons que Bonnefous \& Rakús (1965), Bonnefous (1972) et Soussi (2002) plaçaient cette formation dans le seul Pliensbachien.

Nous nous sommes attachés à étudier systématiquement cette formation afin d'en préciser l'âge. Avant d'y identifier le Sinémurien supérieur (ce travail), nous avions pu dater sa partie supérieure du Pliensbachien inférieur, chronozone à Ibex (Fauré et al. 2007, sous presse; Sekatni-Aich 2010; Sekatni et al. 2013). Nous résumons ces résultats ci-dessous. 


\section{La Formation Zaghouan de la Dorsale tunisienne}

- Le Jebel Ressas est à ce jour le seul dont la Fm Zaghouan ait livré des ammonites dans sa partie moyenne. Rappelons que deux niveaux ont successivement livré des ammonites de la partie supérieure de la sous-chronozone à Stellare (chronozone à Obtusum) et de la partie moyenne de la chronozone à Raricostatum, passage entre les sous-chronozones à Raricostatum et à Macdonnelli.

- Le Jebel Zaghouan, expose $50 \mathrm{~m}$ de calcaires à silex noirs en bancs ondulés de 20 à $50 \mathrm{~cm}$ d'épaisseur, au faciès identique, affleurant particulièrement sur les pentes orientales du massif du Poste optique, à l'est de l'émetteur de télécommunication, où se situe la coupe de référence de la formation (Fauré \& Peybernès 1986a, b). À sa partie supérieure, les ammonites Metaderoceras gr. evolutum (Fucini, 1924) et Reynesocoeloceras sp., permettent d'identifier le Pliensbachien inférieur (chronozone à Ibex) (Fauré et al. 2007). Une discontinuité plane, nette, la sépare de la Fm Bou Gabrine sus-jacente.

- Le Jebel Bou Kornine d'Hammam-Lif exprime deux faciès de la formation:

À l'état stratifié, la formation affleure dans le massif du Poste optique, avec des faciès encore identiques, entre deux discontinuités sédimentaires nettes. Il s'agit de $63 \mathrm{~m}$ de calcaires en bancs compacts, à surface ondulée, séparés par des joints argileux, particulièrement riches en lits de volumineuses chailles sombres (Sekatni-Aich 2010). Le Lytoceras d'affinité sinémurienne L. gr. celticum Geyer, 1886 provient de sa partie supérieure (Sekatni-Aich 2010). Bonnefous \& Rakús (1965) y avaient récolté «Uptonia sp. » [= Metaderoceras gr. evolutum (Fucini, 1924) in coll. ONM, Tunis] de la chronozone à Ibex.

Dans le "Conglomérat inférieur», qui affleure largement dans la partie orientale du djebel, sous forme d'éléments de calcaire à silex remaniés et resédimentés dans une mégabrèche mise en place au Bajocien supérieur (Bonnefous \& Rakús 1965) par effondrement de la plate-forme située en marge du Sillon tunisien (Sekatni et al. 2013). Ces blocs nous ont fourni les ammonites de la chronozone à Ibex (Pliensbachien inférieur) Metaderoceras gr. gemmellaroi (Levi, 1896), Reynesocoeloceras indunense (Meneghini, 1874), Tropidoceras sp. gr. mediterraneum (Gemmellaro, 1884) et T. cf. zitteli (Fucini, 1899) (Sekatni et al. 2013: pl. 1, fig. 1-4), ainsi que de nombreux brachiopodes (Sekatni et al. 2013: pl. 2, fig. 1-3).

\section{La Formation Zaghouan en Tunisie atlasique septentrionale}

Les dépôts équivalents à la Fm Zaghouan offrent encore une large répartition en Tunisie septentrionale où ils sont reconnus dans de nombreuses écailles jurassiques pré-telliennes (Kamoun et al. 1999):

- Les Jebels Djedidi, Châabane et Tamrhoura, situés au sud-ouest de Tunis, correspondent à des écailles alignées le long de la "Cicatrice de Teboursouk» (Pini \& Salaj 1969) dans lesquelles la Fm Zaghouan, qui ne contient pas ici de lits de silex caractéristiques (= Fm Châabane de Soussi 2002) a fourni dans la grande carrière du Jebel Djedidi de nombreuses ammonites du Pliensbachien, chronozones à Ibex et à Davoei (Peybernès et al. 1996; Rakús \& Guex 2002; Soussi 2002; Fauré et al. sous presse).
- Le Jebel Thuburnic, située plus à l'ouest, montre des calcaires à silex dont le faciès et la position sont identiques, suggérant une identité avec la Fm Zaghouan (Peybernès et al. 1996). Leur attribution au Pliensbachien avait déjà été proposée par Rouvier (1977).

L'âge de la formation Zaghouan, en milieu pélagique, Sinémurien supérieur, chronozone à Obtusum à Pliensbachien inférieur, chronozone à Ibex, est identique à celui des Niveaux condensés du sommet de la Fm Oust qui recouvrent les zones de horst et de plate-forme, confirmant l'équivalence latérale des deux unités lithostratigraphiques.

\section{ÉTUDE SYSTÉMATIQUE DES AMMONITES SINÉMURIENNES DU JEBEL RESSAS}

Classe CEPHALOPODA Cuvier, 1795

Sous-classe AMMONOIDEA Zittel, 1884

Ordre PHYLLOCERATIDA Arkell, 1950

Super-famille PHYLLOCERATOIDEA Zittel, 1884

Famille JURAPHYLLITIDAE Arkell, 1950

Genre Juraphyllites Müller, 1939

ESPÈCE TYPE. - Phylloceras diopsis Gemmellaro, 1884 par désignation originale.

\section{Juraphyllites aff. nardii (Meneghini, 1853)}

(Fig. 7B)

aff. Ammonites nardii Meneghini, 1853: 27.

aff. Rhacophyllites nardii - Fucini 1901: pl. 7, fig. 1-7 (figuration de l'holotype).

aff. Juraphyllites nardii - Meister \& Böhm 1993: pl. 2, fig. 8, avec synonymie. — Dommergues et al. 1994: pl. 1, fig. 5-6; 1995: pl. 1, fig. 6, avec synonymie. — Blau 1998: pl. 1, fig. 7-16. — Meister \& Friebe 2003: 24, avec synonymie. — Géczy \& Meister 2007: pl. 6, fig. 1-3 avec synonymie. - Meister et al. 2017: pl. 16, fig. 5.

aff. Juraphyllites aff. ? nardii - Dommergues et al. 2008: fig. 4A, B. aff. Juraphyllites (Juraphyllites) nardii - Dommergues \& Meister 2017: 200, fig. 9 .

MAtÉRIEl EXAMiné. - Jebel Ressas, Paroi Rouge, niv. 21.1 ex. (PFT 207).

\section{DESCRIPTION}

Ce Juraphyllites très mal conservé, à la coquille usée et aplatie, est rapproché de J. nardii avec lequel il partage un enroulement semi-involute et des flancs hauts, portant sur toute leur hauteur une costulation rigide qui prend du relief vers leur partie haute. Le rebord ombilical est bien marqué.

\section{ÂGE ET RÉPARTITION}

L'espèce provient de la partie moyenne de la chronozone à Raricostatum où elle est récoltée dans le même banc que Paltechioceras bavaricum (Böse, 1894) et $P$. cf. charpentieri (Schafhäult, 1847) (sous-chronozones à Rariscostatum à Macdonnelli pars). 
Il s'agit d'une espèce purement téthysienne, à l'extension stratigraphique large, étendue du Sinémurien supérieur à la base du Pliensbachien. Sur la marge nord de la Téthys, elle est présente de la partie inférieure de la chronozone à Obtusum (Meister \& Böhm 1993; Meister \& Friebe 2003) à la chronozone à Raricostatum (Meister et al. 2017). Elle est également connue dans les régions pacifiques (Palfy 1991).

\section{Ordre PSILOCERATIDA Housa, 1965 \\ Superfamille ARIETITOIDEA Hyatt, 1875 \\ Famille ARIETITIDAE Hyatt, 1875 \\ Sous-famille ARnioceratinAE Spath, 1924}

Genre Arnioceras Hyatt, 1867

ESPÈCE TYPE. - Arnioceras cuneiforme Hyatt, 1867 par désignation subséquente (Arkell 1951: 217).

\section{Arnioceras gr. ceratitoides (Quenstedt, 1849) rejectum Fucini, 1902}

(Fig. 7C1-C3)

Ammonites ceratitoides Quenstedt, 1849: pl. 19, fig. 13.

Arnioceras rejectum Fucini, 1902: pl. 14, fig. 12-14; pl. 16, fig. 1-6.

Arnioceras ceratitoides - Meister \& Böhm 1993: pl. 4, fig. 3, 5, 6, 10 , avec synonymie.

Arnioceras gr. rejectum - El Hariri et al. 2010: 232, avec synonymie.

Matériel eXaminé. - Jebel Ressas, Paroi Rouge, niv. 20. 1 ex. (PFT201).

\section{DESCRIPTION}

Malgré son mauvais état de conservation, cet Arnioceras de petite taille est bien caractérisé par un stade lisse court, n'excédant pas $5 \mathrm{~mm}$ de longueur, auquel succède une costulation de densité moyenne faite de côtes tranchantes au tracé tendu, très en relief au rebord ventral, avant leur projection ventrale très courte (Fig. 3a). La section est subrectangulaire, comprimée, et l'enroulement moyennement évolute. La carène, peu élevée, est bordée de méplats creusés de sillons bien marqués mais peu profonds (Fig. 3b).

\section{DisCUSSION}

Les Arnioceras du Sinémurien supérieur téthysien sont un groupe très variable, en termes de densité de costulation et de section du tour, dont le large spectre morphologique est très bien illustré par la multiplication des «espèces » de Fucini (1902). Ces formes ont longtemps été regroupées sous le taxon de $A$. ceratitoides, qui est une espèce d'Adneth figurée par Quenstedt (1849) et plusieurs fois interprétée par les auteurs (Schlegelmilch 1992; Cassinis \& Cantaluppi 1967; Cantaluppi \& Corti 1969; Blau 1998; Géczy \& Meister 2007). Constatant que les âges et les provenances de tous les spécimens figurés par Fucini étaient à peu près identiques, Dommergues et al. (1994) les rapportent à une seule entité spécifique très variable dont la forme moyenne est $A$. rejectum. Il est très vraisemblable que $A$. ceratitoides et $A$. rejectum ne forment qu'une seule et même espèce, dans le spectre de laquelle nous incluons notre spécimen.

\section{ÂGE ET RÉPARTITION}

Récolté avec Asteroceras gr. saltriensis (Parona, 1896) et $A$. cf. meridionalis Dommergues, Meister \& Mettraux, 1990, notre spécimen provient clairement de la partie terminale de la sous-chronozone à Stellare.

Les Arnioceras du groupe de Arnioceras ceratitoides-rejectum sont souvent cités dans le Sinémurien supérieur de la région méditerranéenne où ils caractérisent une large plage de temps. Ils recouvrent toute la durée de la chronozone à Obtusum dans l'Austroalpin (Meister \& Böhm 1993; Dommergues et al. 1995 ; Meister \& Friebe 2003), les Apennins (Dommergues et al. 1994) et les Chaînes Bétiques (Braga et al. 1985), la seule sous-chronozone à Stellare dans les Alpes calcaires méridionales (Meister et al. 2017) et la seule partie terminale de la sous-chronozone à Stellare dans les Montagnes de Bakony où, comme en Tunisie, l'espèce accompagne Asteroceras gr. saltriensis (Géczy \& Meister 2007).

Sur la marge maghrébine de la Téthys, le groupe est bien représenté dans le Rif (Mouterde 1965) et dans le Haut-Atlas marocain (de Midelt, de Beni Melal et de Rich) (El Hariri et al. 1996, 2010; Lachkar et al. 1998; Guex et al. 2008) où l'extension stratigraphique de Arnioceras gr. rejectum est plus large car l'espèce précède le plus souvent les couches à Asteroceras avant de s'associer à lui dans la sous-chronozone à Stellare, voire dans des niveaux élevés de la sous-chronozone (Lachkar et al. 1998).

Il est singulier qu'aucun exemplaire d'Arnioceras de ce groupe n'ait été à ce jour signalé dans le Sinémurien supérieur de Tunisie.

\section{Sous-famille AsterocerATINAE Spath, 1946}

Genre Asteroceras Hyatt, 1867

ESPÈCE TYPE. - Ammonites stellaris J. Sowerby, 1815, par désignation subséquente (Buckman 1911).

\section{Asteroceras gr. saltriensis (Parona, 1896)}

$$
\text { (Fig. 6A, B) }
$$

Arietites saltriensis Parona, 1896: pl. 8, fig. 2, 3.

Asteroceras saltriense - Fucini 1903: pl. 33, fig. 4-6. — ? Palfy 1991: pl. 10, fig. 9.

Asteroceras obtusum - Pozzi 1960: pl. 42, fig. 1-2. - Sacchi-Vialli \& Cantaluppi 1961: pl. 3, fig. 3, 4. — ? Rakús 1994: pl. 5, fig. 3.

? Asteroceras sp. gr. stellare - Sacchi-Vialli \& Cantaluppi 1961: pl. 3, fig. 6 seule.

? Asteroceras saltriense - Palfy \& Smith 1994: pl. 1, fig. 1 .

Asteroceras aff. saltriense - Dommergues et al.1995: pl. 5, fig. 11 . 
? "Asteroceras" aff. blakei - Alkaya \& Meister 1995: pl. 4, fig. 2, 4.

Asteroceras cf. saltriense - Johanson et al. 1997: pl. 1, fig. 5.

Asteroceras gr. saltriensis - Meister \& Friebe 2003: pl. 6, fig. 1; pl. 7, figs 1, 3; pl. 8, fig. 1. — Géczy \& Meister 2007: pl. 18, figs 1, 2; pl. 19 , figs 1,4 ; pl. 20, figs 3,4 .

Matériel eXaminé. - Jebel Ressas, Paroi Rouge, niv. 20. 1 ex. partiellement prélevé, PF202 et 1 ex. non prélevé.

\section{DESCRIPTION}

Deux spécimens mal conservés, l'un partiellement prélevé (Fig. 6A), le second n'ayant pu être prélevé (Fig. 6B), sont rapprochés de cette espèce à la coquille assez nettement évolute pour un Asteroceras (O/D = 42-43\%). Les tours externes sont caractérisés par une section ogivale, près de deux fois plus haute que large, des flancs bombés et l'absence des sillons péricarénaux caractéristiques du genre, au profit de simples méplats obliques. Par ailleurs, l'aire ventrale est arrondie à tectiforme, axée par une carène émoussée, totalement absente après $120 \mathrm{~mm}$ de diamètre. Les côtes sont fortes et rigides sur le flanc et leur relief tend à s'estomper dans le tiers supérieur du flanc, au niveau de leur légère inflexion vers l'avant. Dans les tours internes l'ornementation est beaucoup plus grossière, avec des côtes fortes et rigides gardant un important relief sur toute la hauteur du tour.

\section{DisCUSSION}

A. saltriensis, appartient à un groupe d'Asteroceras évolués qui, tous, montrent une même tendance à l'affaiblissement des sillons qui bordent la carène, et dont l'espèce la mieux documentée est Asteroceras blakei Spath, 1925. Cette morphologie suggère une transition vers le genre Aegasteroceras Spath, 1925. Au sein de ce groupe, $A$. meridionalis Dommergues, Meister \& Mettraux, 1990 est l'espèce la plus proche de $A$. saltriensis, mais sa coquille est plus comprimée et plus nettement involute. Les taxons méditerranéens A. obtusum (Sowerby, 1817) sensu Sacchi-Vialli \& Cantaluppi, 1961 et Asteroceras obtusum (Sowerby, 1817) sensu Pozzi, 1960 s'avèrent tous très proches, voire identiques à $A$. saltriensis.

\section{ÂGE ET RÉPARTITION}

Dans la région ouest-téthysienne, $A$. saltriensis est une espèce de la sous-chronozone à Stellare (Meister \& Friebe 2003) et, comme Asteroceras blakei et $A$. meridionalis sur les platesformes ouest européennes (Dommergues 1993; Edmunds et al. 2016), elle n'est présente que dans la partie supérieure de la sous-chronozone, ce qui est très vraisemblablement la position stratigraphique de nos spécimens dans le Jebel Ressas.

A. saltriensis offre une répartition purement méditerranéenne et est signalé dans les Alpes calcaires italiennes (Parona 1896; Sacchi-Vialli \& Cantaluppi 1961), l'Austro-Alpin supérieur autrichien (Dommergues et al. 1995; Meister \& Friebe 2003) et italien (Pozzi 1960) et les Monts du Bakony hongrois (Géczy \& Meister 2007). Elle n’avait jamais été citée à ce jour dans la Dorsale tunisienne ni sur la marge nord-africaine de la Téthys. A. saltriensis est également fréquemment cité dans la région pacifique (Palfy 1991).

\section{Asteroceras cf. meridionalis \\ Dommergues, Meister \& Mettraux, 1990}

(Fig. 7D)

cf. Asteroceras meridionalis Dommergues, Meister \& Mettraux, 1990: pl. 2, figs 2-9; pl. 3, fig. 1. — Dommergues et al. 2005: pl. 1, figs 3-6.

Asteroceras cf. suevicum - Braga et al. 1985: pl. 1, fig. 5. - Blau 1998: pl. 2, figs 13-16; pl. 14, fig. 6.

Asteroceras aff. suevicum - Corna et al. 1997: pl. 10, fig. 1. Meister \& Friebe 2003: 32.

Aegasteroceas aff. sagittarium - Guex et al. 2008: pl. 8, fig. 1.

MatérIEL eXAminé. — Jebel Ressas, Paroi Rouge, niv. 20. 1 ex. (PFT 203).

\section{DESCRIPTION}

Cet Asteroceras, dont nous ne disposons que d'un unique fragment de petite taille, est toutefois bien caractérisé par sa section ogivale comprimée, son enroulement semi-involute, ses flancs bombés régulièrement convergents vers une aire ventrale étroite dont la forte carène est bordée par deux sillons bien marqués. Les côtes sont fortes, espacées, régulièrement arquées vers l'avant. Leur relief, maximum au-dessus du rebord ombilical, s'atténue progressivement en direction du rebord latéro-ventral qu'elles atteignent toujours nettement au niveau d'une pseudo-carène. L'écrasement de notre spécimen ne permet cependant pas de vérifier l'identité totale avec $A$. meridionalis dont l'ombilic semble légèrement plus fermé et la coquille moins comprimée.

\section{RAPPORTS ET DIFFÉRENCES}

À taille identique, $A$. cf. meridionalis est plus involute et plus comprimé que $A$. saltriensis dont la costulation est plus rigide et plus grossière, bien marquée sur toute la hauteur du flanc. A contrario, les Asteroceras du groupe de A. varians Fucini, 1903 s'en démarquent aisément par l'effacement progressif des côtes qui n'atteignent pas le rebord ventral. A. suevicum (Quenstedt, 1884) présente des flancs plus hauts et plus plats et sa costulation est plus fine et plus rigide.

\section{DISCUSSION}

A. cf. meridionalis appartient, comme $A$. gr. saltriensis, à un groupe d'Asteroceratinae tardifs caractérisés par l'effacement progressif des sillons péricarénaux et dont le chef de file est $A$. blakei Spath, 1925. Mais, à taille égale, cette dernière espèce présente cependant une section et une aire ventrale plus larges et plus arrondies et des sillons péri-carénaux moins bien marqués. Nous retrouvons les mêmes caractères ornementaux chez plusieurs spécimens méditerranéens décrits sous le taxon de Asteroceras suevicum (Quenstedt, 1884), par exemple, Asteroceras cf. suevicum sensu Braga et al. (1985) et Asteroceras aff. suevicum sensu Blau (1998), ainsi que chez Aegasteroceas aff. sagittarium (Blake, 1876) sensu Guex et al. (2008) qui est vraisemblablement identique à notre spécimen.

\section{ÂGE ET RÉPARTITION}

A. meridionalis est attribué à la partie supérieure de la souschronozone à Stellare par Dommergues et al. (2005), ce qui est en accord avec son association avec $A$. gr. saltriensis dans la Fm Zaghouan du Jebel Ressas. Cette espèce, décrite au sein 

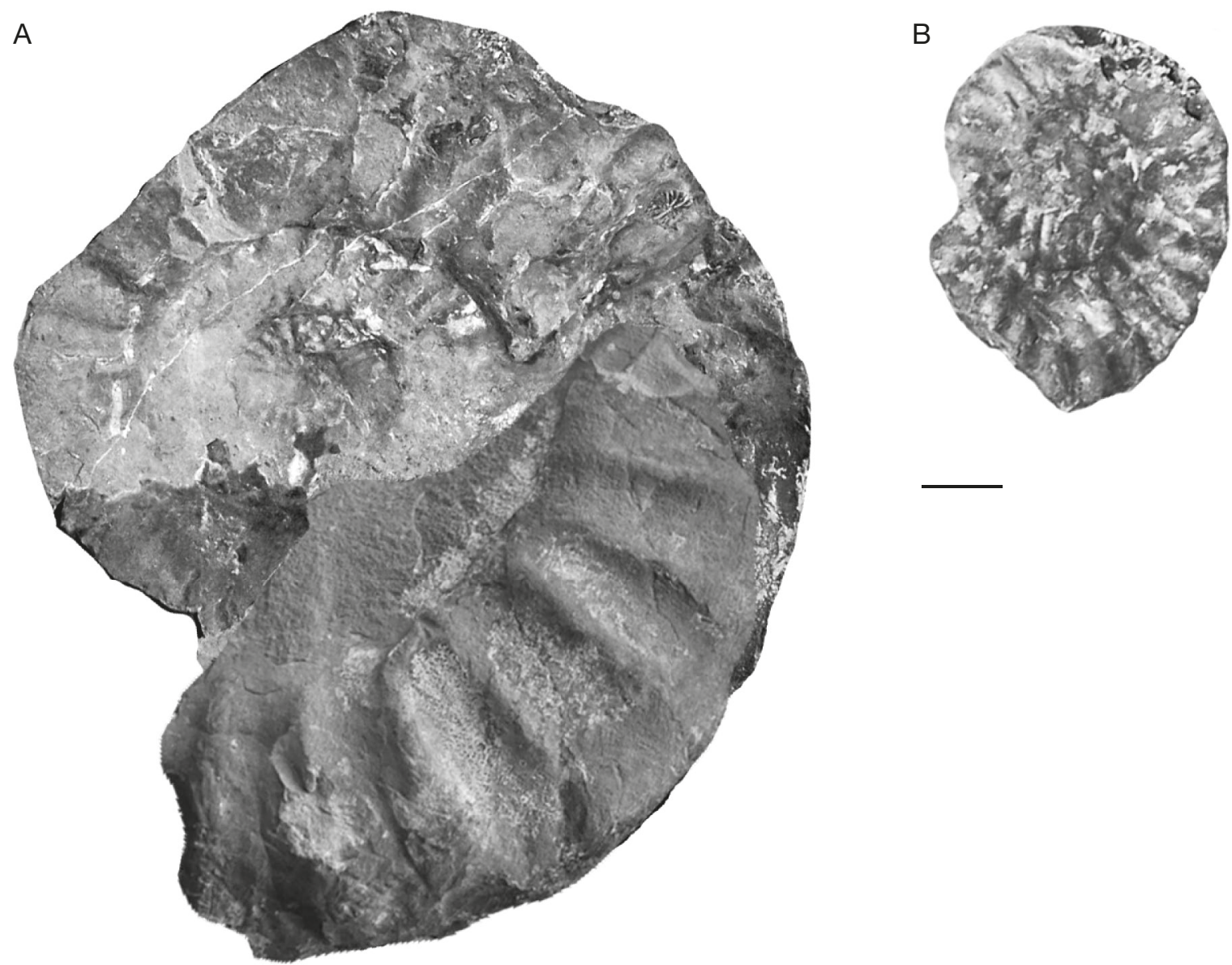

FIG. 6. - A, B, Asteroceras gr. saltriensis (Parona, 1896), Sinémurien, chronozone à Obtusum, sous-chronozone à Stellare, Paroi Rouge, niv. 20: A, photomontage superposant l'image du fragment prélevé (PFT202) à la photographie du spécimen non extirpable, resté in situ; B, photographie de terrain d'un spécimen non extirpable et non prélevé. Échelle: $1 \mathrm{~cm}$.

d'une abondante population des Alpes occidentales suisses (Dommergues et al. 1990) et françaises (Oisans, Zone subbrianconnaise) (Corna et al. 1997; Dommergues et al. 2005), n'était pas identifiée avec certitude en dehors des confins méridionaux de la Province nord-ouest européenne. Mais nous lui attribuons des formes synonymes, communes dans la région méditerranéenne proprement dite comme l'Austroalpin autrichien (Blau 1998), les Cordillères Bétiques (Braga et al. 1985) et le Haut-Atlas marocain (Guex et al. 2008).

Genre Epophioceras Spath, 1924

ESPÈCE TYPE. - Ammonites landrioti d'Orbigny, 1849 par désignation originale.

\section{Epophioceras aff. landrioti (d'Orbigny, 1850)}

(Fig. 7A1-A3)

aff. Ammonites landrioti d'Orbigny, 1850: 213. — Reynès 1879: pl. 29, fig. 1-5. — Thévenin 1907: pl. 7, figs 4-5.

aff. Vermiceras landrioti - Fucini 1902: pl. 7, fig. 1, 2.

aff. ? Vermiceras affine Fucini, 1902: pl. 14, fig. 6. aff. ? Tmaegophioceras gr. laeve - Mouterde et al. 1986: pl. 2, fig. 4.

aff. Epophioceras landrioti - Guérin-Franiatte 1966: pls 217, 218. Schlegelmilch 1992: pl. 20, fig. 2. - Braga et al. 1985: pl. 1, fig. 1. - Blau 1998: pl. 2, fig. 12. - Howarth 2002: 125.

aff. Epophioceras gr. landrioti - Meister \& Friebe 2003: pl. 9, fig. 4.

aff. ? Epophioceras sp. - Edmunds et al. 2016: fig. 14a.

aff. ? Epophioceras sp. - Meister et al. 2017: pl. 5, fig. 3.

aff. ? Epophioceras aff. landrioti - Dommergues \& Meister 2017: fig. 85.

Matériel eXaminé. - Jebel Ressas, Paroi Rouge, niv. 20. 3 ex. (PFT204, 205, 206).

\section{DESCRIPTION}

Avec son enroulement serpenticône caractéristique, nous incluons dans le genre Epophioceras ce tour externe d'un spécimen de $10 \mathrm{~cm}$ de diamètre. Nous l'avons rapproché de l'espèce E. landrioti en raison de la relative faible densité de sa costulation qui, comme chez cette espèce, tend à s'effacer progressivement vers $9-10 \mathrm{~cm}$ de diamètre. Avant ce diamètre, sa costulation est particulièrement grossière pour le genre. Elle est faite de côtes fortes, d'allure pincée, séparées par des espaces concaves 
plus larges qu'elles (Fig. 1a). Leur relief s'accentue jusqu'au deux tiers de la hauteur du tour, puis il s'atténue rapidement sur l'arrondi ventro-latéral, après une discrète projection vers l'avant. La section est subquadratique à subcirculaire, à peine plus haute que large, avec des flancs légèrement bombés et une aire ventrale large et convexe, axée par une carène émoussée, peu visible, bordée par deux méplats inclinés, plats à très légèrement concaves. Après $80 \mathrm{~mm}$ de diamètre, le relief de l'ornementation latérale s'atténue progressivement, sans avoir atteint de stade lisse à $100 \mathrm{~mm}$ de diamètre.

\section{DisCUSSION}

Avec la costulation grossière, c'est le spécimen nord-ouest européen figuré par Schlegelmilch (1992) qui illustre le mieux le style ornemental de notre échantillon. Par ailleurs, les populations européennes de l'espèce (Guérin-Franiatte 1966) affichent le plus souvent une plus grande taille, un enroulement sensiblement plus serpenticône et une costulation globalement moins grossière, ne permettant pas une identification exacte de notre individu au type de d'Orbigny.

Parmi les formes téthysiennes, les spécimens austro-alpins figurés par Blau (1998) et Meister \& Friebe (2003) sont caractérisés par une costulation plus grossière et une moindre tendance à l'effacement de l'ornementation. Notre spécimen peut aussi être comparé à des tours internes de E. cognitum Guérin-Franiatte, 1966 et d'espèces vraisemblablement synonymes, E. hebetius Guérin-Franiatte, 1966 et E. asperum Guérin-Franiatte, 1966, mais ces formes ne montrent aucune tendance à l'effacement de la costulation, leur section est moins comprimée et leur aire ventrale est plus aplatie, portant une carène plus forte, bordée par deux sillons plus marqués. L'exagération de tels caractères est évidente chez E. deciduum (Hyatt, 1867), espèce souvent placée dans le genre Echioceras (Getty 1973) et dont l'appartenance au genre Epophioceras est encore discutée (Dommergues et al. 1995).

A l'inverse, les formes méditerranéennes décrites dans les Apennins (Fucini 1902), dans le Rif (Mouterde et al. 1986) et dans les Chaînes Bétiques (Braga et al. 1985) montrent une costulation plus dense, au relief plus atténué tout au long de l'ontogénèse, avec apparition d'un stade totalement lisse. Une même tendance à l'atténuation rapide de l'ornementation est retrouvée chez l'espèce européenne E. bochardi (Reynès, 1879).

E. longicella (Quenstedt, 1885) est toujours plus densément costé, sa section plus large et sa carène encadrée de deux sillons bien marqués.

\section{ÂGE ET RÉPARTITION}

L'association de notre spécimen avec Asteroceras gr. saltriensis et $A$. cf. meridionalis, le place clairement dans la partie supérieure de la sous-chronozone à Stellare.
E. landrioti est une forme ubiquiste, peu abondante à la fois dans les Provinces ouest-européenne et méditerranéenne. En raison de sa rareté, les citations stratigraphiquement bien documentées sont peu nombreuses. Sur les plates-forme ouesteuropéennes, l'espèce nominale est rapportée à la chronozone à Obtusum en Lorraine (Guérin-Franiatte 1966), dans le Jura méridional (Corna 1987), en Allemagne méridionale (Schlegelmilch 1992) et en Angleterre (Page 2003) où son âge est précisé à la sous-chronozone à Stellare (Howarth 2002), voire à sa partie supérieure (Edmunds et al. 2016). Dans les dépendances méditerranéennes du Domaine téthysien, l'espèce est signalée dans la chronozone à Obtusum de l'Austroalpin supérieur (Blau 1998) où Meister \& Friebe (2003) la placent dans la sous-chronozone à Stellare, ce qui s'accorde aussi avec l'âge de notre spécimen. Dans les Cordillères Bétiques (Braga et al. 1985), elle coexiste avec des Asteroceras que nous regroupons ici avec $A$. meridionalis Dommergues et al., 1990 dont nous avons vu que la morphologie évoquait aussi un niveau élevé de la sous-chronozone à Stellare.

Famille ECHIOCERATIDAE Buckman, 1913

Genre Paltechioceras Buckman, 1924

ESPÈCE TYPE. - Paltechioceras elicitum Buckman, 1924 par désignation originale.

Paltechioceras bavaricum (Böse, 1894)

(Fig. 7E1, E2, F, G, H, I)

Arietites bavaricus Böse, 1894: pl. 56, fig. 1, 2.

Arietites cf. conybeari - Hug 1899: pl. 12, figs 3-4.

Echioceras subquaratum Buckman, 1914: 96c.

Euechioceras bavaricum - Trueman \& Williams 1925: 726.

Paltechioceras bavaricum - Donovan 1958: pl. 4, fig. 2a, b, avec synonymie. - Topchishvili et al. 2006: pl. 6, fig. 6 ?; pl. 7, fig. 1 .

non Paltechioceras cf. bavaricum - Suarez-Vega 1974: pl. 6, fig. 1. (= P. tardecrescens (Hauer, 1856)).

non Paltechioceras gr. bavaricum - Rakús \& Guex 2002: pl. 13, fig. 6, 10 (= P. cf. tardecrescens (Hauer, 1856)).

Matériel eXaminé. - Jebel Ressas, Paroi Rouge, niv. 21. 5 ex. (PFT 208, 209, 210, 211, 212).

\section{DESCRIPTION}

Plusieurs spécimens provenant d'un niveau identique correspondent parfaitement à la description originale de cette

FIG. 7. - A1-A3, Epophioceras aff. landrioti (d'Orbigny, 1850), Sinémurien, chronozone à Obtusum, sous-chronozone à Stellare, Paroi Rouge, niv. 20, PFT204: B, Juraphyllites aff. nardii (Meneghini, 1853), chronozone à Raricostatum, Paroi Rouge, niv. 21, PFT207; C1-C3, Arnioceras gr. ceratitoides (Quenstedt, 1849) rejectum Fucini, 1902, chronozone à Obtusum, sous-chronozone à Stellare, Paroi Rouge, niv. 20, PFT 201; D, Asteroceras cf. meridionalis Dommergues, Meister \& Mettraux, 1990, chronozone à Obtusum, sous-chronozone à Stellare, Paroi Rouge, niv. 20, PFT 203; E, F, G, H, I, Paltechioceras bavaricum (Böse, 1894), chronozone à Raricostatum, Paroi Rouge, niv. 21: E1-E2, PFT208; F, PFT209; G, PFT210; H, PFT211; I, PFT212; J, K, L, Paltechioceras cf. charpentieri (Schafhäult, 1847), chronozone à Raricostatum, Paroi Rouge, niv. 21: J, PFT213; K, PFT214; L, PFT215. Le matériel appartient à la collection Ph. Fauré. II est déposé dans les collections de l'Office national des Mines de Tunis (ONM). Échelle: $1 \mathrm{~cm}$ (grandeur naturelle). 


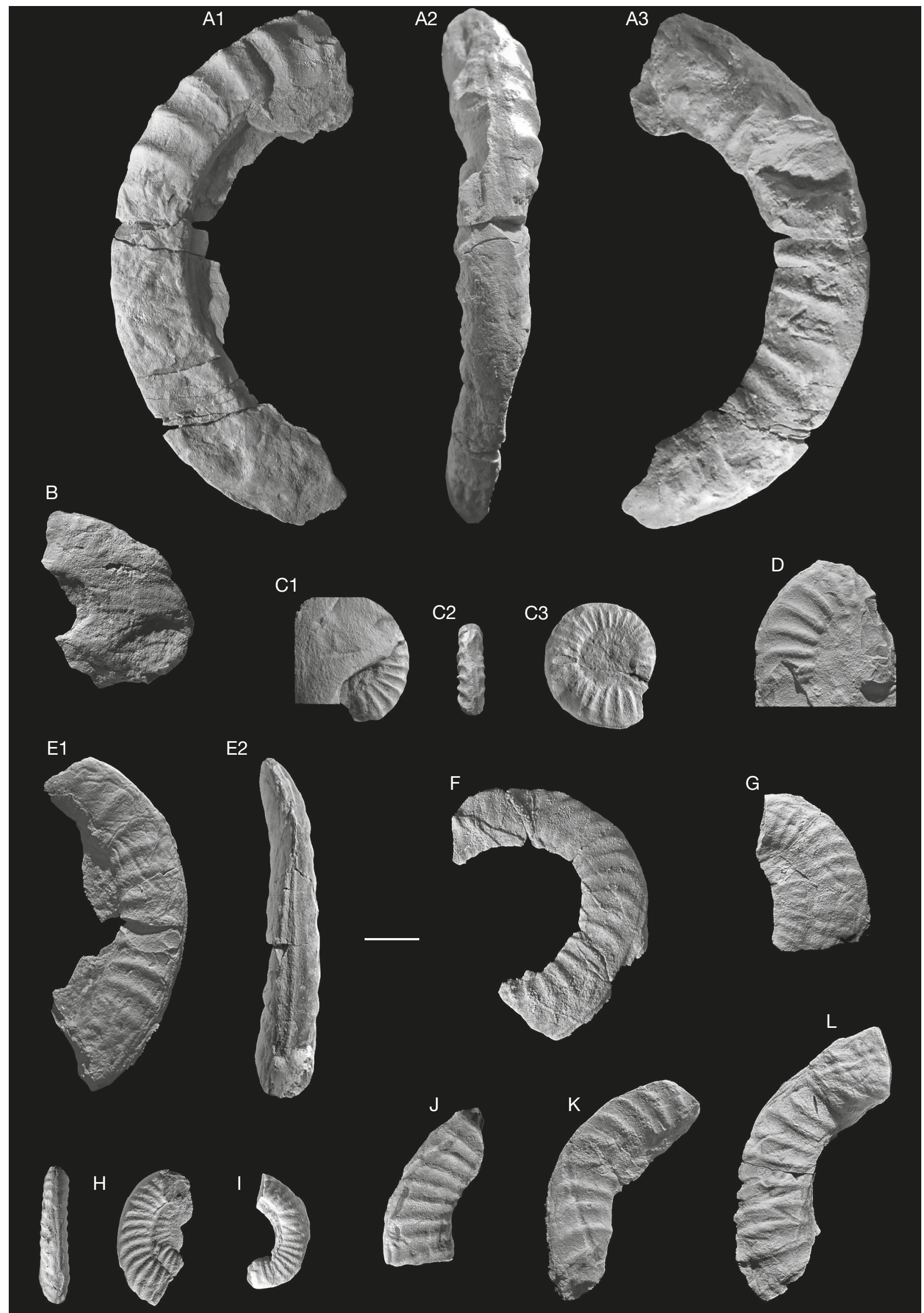


espèce peu connue (lectotype désigné par Donovan 1958). La caractéristique principale de cette espèce est de posséder une costulation lâche, pour un Paltechioceras, et des côtes proverses, au relief relativement émoussé, régulièrement arquées vers l'avant où elles rejoignent une pseudo-carène assez bien marquée située sur l'angle ventro-latéral. Comme sur la figure de Böse (1894), l'enroulement est subserpenticône, la section du tour subelliptique, nettement comprimée, plus haute que large et les flancs hauts, subaplatis. L'aire ventrale est étroite et la carène est bordée de deux sillons bien marqués, quelle que soit la taille de l'individu, avec l'ébauche d'une tricarénation.

Malgré l'écrasement de leur coquille, les deux spécimens Fig. 7F, G montrent un même style de costulation et sont rapportés à cette espèce, bien que leur coquille semble, vraisemblablement artéfactuellement, plus involute. Il est possible d'y percevoir la présence de sillons péricarénaux.

\section{RAPPORT ET DIFFERENCE}

Paltechioceras charpentieri (Hug, 1899) est certainement l'espèce la plus proche de $P$. bavaricum et seuls des détails morphologiques permettent de séparer les deux taxons qui ne diffèrent que par une section moins comprimée chez $P$. charpentieri et une costulation faite de côtes rectiradiées, rigides, au relief plus marqué.

P. rothpletzi (lectotype figuré par Meister et al. 2009) est une espèce proche de $P$. bavaricum qui s'en sépare pour une costulation plus dense tout au long de l'ontogénèse, avec laquelle elle partage de nombreux caractères morphologiques (côtes proverses, arquées vers l'avant; coquille relativement involute pour un Paltechioceras; section comprimée plus haute que large, sillons péri-carénaux bien marqués avec ou sans tricarénation...).

\section{ÂGE}

$P$. bavaricum et $P$. rothpletzi appartiennent à un même groupe de Paltechioceras primitifs, très répandus sur les deux marges de la Téthys méditerranéenne que Blau (1998) et Blau \& Meister (2000) placent dans la partie moyenne de la chronozone à Raricostatum, à la partie supérieure de la sous-chronozone à Raricostatum où ils précèdent l'apparition du genre Leptechioceras (horizon à Liciense de Blau \& Meister 2000). C'est très vraisemblablement l'âge de $P$. bavaricum en Tunisie.

A noter que $P$. gr. bavaricum, figuré par Rakús \& Guex (2002: pl. 13, fig. 6, 10) dans les niveaux condensés de la Dorsale tunisienne appartient au groupe de $P$. tardecrescens (Dommergues \& Meister 2017) où il caractérise la partie terminale de la chronozone à Raricostatum.

\section{RÉPARTITION}

Depuis la description de Böse (1894), force est de constater qu'aucun spécimen incontestable de $P$. bavaricum n'a à ce jour été figuré. D’après les citations les plus fidèles de l'espèce, $P$. bavaricum serait surtout représenté de part et d'autre de la marge nord de la Téthys méditerranéenne, sur son versant européen (Préalpes médianes) et plus largement, sur son versant téthysien (Austroalpin allemand et autrichien, Géor- gie). L'absence de figuration ne permet pas de confirmer les citations de l'espèce dans les Apennins (Cecca et al. 1987: 72), ni dans les Cordillères Bétiques (Braga et al. 1985). La présence du groupe de $P$. bavaricum-rothpletzi est par ailleurs bien documentée dans le Haut-Atlas (Guex et al. 2008) et dans les Régions Circum-Pacifiques comme le Mexique (Sierra Madre orientale, Blau et al. 2003; Meister et al. 2009), la Colombie Britannique (Palfy 1991), le Nevada et l'Oregon (Smith 1981).

\section{Paltechioceras cf. charpentieri (Schafhäult, 1847)} (Fig. 7J, K, L)

cf. Ammonites charpentieri Schafhäult, 1847: 810; 1851: pl. 16, fig. 22, seule.

cf. Leptechioceras charpentieri - Getty 1973: pl. 2, figs 6, (cf.) 1, 4.

cf. Paltechioceras charpentieri - Blau 1998: pl. 6, figs 2, (cf.) 1, 4, avec synonymie. - Meister \& Friebe 2003: fig. 11, figs 4, 6.

cf. Paltechioceras cf. charpentieri - Fauré 2002: pl. 2, figs 12, 13, 14a, b. — Fauré \& Alméras 2006: pl., figs 15-18.

cf. Paltechioceras gr. charpentieri-Géczy \& Meister 2007: pl. 30, fig. 3.

Matériel eXAminé. - Jebel Ressas, Paroi Rouge, niv. 21. 4 ex. (PFT213, 214, 215, 216).

\section{DESCRIPTION}

Plusieurs fragments d'un Echioceratidae sont ici rapprochés de Paltechioceras charpentieri en raison de leur costulation forte, faite de côtes au relief bien marqué, à la fois proverses et légèrement arquées vers l'avant, s’infléchissant légèrement sur le bord externe en perdant de leur relief. Malgré les artéfacts dus à l'écrasement des coquilles, on peut observer que, comme chez cette espèce, la carène est nettement bordée par deux sillons.

\section{DISCUSSION}

P. charpentieri est considéré par Blau (1998) et Meister \& Friebe (2003) comme une espèce intermédiaire entre les deux genres Paltechioceras et Leptechioceras. La présence des sillons péricarénaux permet de placer, sans ambiguïté, nos spécimens dans le genre Paltechioceras.

\section{ÂGE ET RÉPARTITION}

P. charpentieri est surtout connu sur la marge nord de la Téthys (Austroalpin supérieur d'Autriche et de Hongrie), où il est récolté in situ dans la partie inférieure de la sous-chronozone à Macdonnelli (Horizon à Meigeni-Charpentieri de Blau \& Meister 2000). Son âge est identique dans les confins méridionaux des plates-formes européennes (Pyrénées, Corbières). Il est très vraisemblable que le niveau stratigraphique de nos spécimens en soit très proche, comme il est très certainement très proche de celui de $P$. bavaricum avec lequel il est associé dans un contexte de vraisemblable condensation sédimentaire.

P. charpentieri est pour la première fois signalé sur la marge africaine de la Téthys méditerranéenne. 


\section{LE SINÉMURIEN DE TUNISIE. ESSAI DE CORRÉLATIONS}

Nous discuterons ci-dessous de la place des faunes sinémuriennes recueillies en Tunisie en les situant dans le cadre biochronologique standard zonal et sous-zonal établi en Europe du nord-ouest (Dean et al. 1961; Corna et al. 1997; Page 2003), comparé à des échelles biozonales à valeur plus locale, comme celle établie en domaine nord-téthysien par Blau (1998) et Blau \& Meister (2000) (Fig. 8).

\section{Le Sinémurien de la Formation Zaghouan}

La Fm Zaghouan représente un faciès pélagique du Sinémurien supérieur et du Pliensbachien inférieur, uniquement présent en Tunisie atlasique septentrionale où il est particulièrement bien exprimé dans les jebels septentrionaux de la Dorsale tunisienne (Fig. 1). Elle n'a fourni de faune sinémurienne que dans le Jebel Ressas où deux niveaux d'ammonites ont pu être repérés dans la partie moyenne de la Formation (Fig. 4, 7):

- Le niveau 20: Les Asteroceras (A. gr. saltriensis et $A$. cf. meridionalis) sont des formes évoluées qui appartiennent à un groupe d'espèces caractérisées par l'affaiblissement des sillons qui bordent la carène (e.g. A. blakei Spath, 1925 et $A$. suevicum (Quenstedt, 1884)), fréquentes dans la partie supérieure de la sous-chronozone à Stellare (chronozone à Obtusum).

La présence de $A$. ceratitoides-rejectum et d'Epophioceras aff. landrioti, ne contredit pas cette attribution, ces deux espèces pouvant aussi être présentes dans la sous-chronozone à Stellare (Meister \& Friebe 2003; Géczy \& Meister 2007). Il faut noter qu'aucun spécimen appartenant au genre Parasteroceras n'y a été récolté.

- Le niveau 21 : Paltechioceras bavaricum présente de fortes affinités morphologiques avec les Echioceratidae primitifs (e.g. Paltechioceras gr. rothpletzi) du sommet de la sous-chronozone à Raricostatum (chronozone à Raricostatum) que l'on retrouve dans l'Austroalpin dans l'horizon à Liciense et Rothpletzi de Blau \& Meister (2000) (Fig. 8). P. cf. charpentieri semble légèrement plus récent et se placerait à la base de la sous-chronozone à Macdonnelli, à un niveau correspondant à l'horizon à Meigeni et Charpentieri de Blau \& Meister (2000) (Fig. 8). Un certain degré de condensation sédimentaire, au sein de la Fm Zaghouan, explique très vraisemblablement l'association de ces deux espèces dans le même banc.

La Chronozone à Ibex (zone à Demonense de Braga et al. 1982) est identifiée, dans la partie supérieure de la formation, dans les Jebels Zaghouan, Bou Kornine de Hammam-Lif et Djedidi, avec Metaderoceras gr. evolutum, M. gemmellaroi, Reynesocoeloceras sp., Tropidoceras sp. gr. mediterraneum et T. cf. zitteli (Sekatni et al. 2013).

\section{Le SinÉmurien des Niveaux CONDENSÉS \\ DU SOMMET DE LA FM OUST}

Rappelons que cette unité n'est présente que dans les seuls jebels centraux de la Dorsale tunisienne. Située au sommet de la Fm Oust, elle se présente, sur 0,5 à $1 \mathrm{~m}$ d'épaisseur, sous la forme d'une succession de 4 à 5 bancs de calcaire condensé, remanié, glauconieux, séparés par des hard-grounds. Ces bancs, déposés dans un contexte d'instabilité sédimentaire, sont discontinus, lenticulaires, parfois dédoublés, toujours séparés par des périodes de lacune sédimentaire plus ou moins longues, ce qui explique les importantes variations latérales, parfois rapides, entre les profils décrits par les auteurs.

Leur riche faune d'ammonites du Pliensbachien est pour la première fois décrite par Dubar (1953) dans le Jebel Bent Saïdane. Elle est ensuite successivement étudiée par Rakús \& Biely (1970), Fauré \& Peybernès (1986a, b), Dommergues et al. (1986) et Rakús \& Guex (2002) qui y décrivent de très nombreuses ammonites dont les âges s'échelonnent cette fois du Sinémurien inférieur (sommital) au Pliensbachien inférieur. La confrontation de toutes ces études nous permet de distinguer six assemblages fauniques distincts:

- Les assemblages 1 et 4, à faune sinémurienne, exprimés dans le Jebel Oust (sud-est du Jebel, Carrière romaine) et dans le Grand Zaghouan (Attaque Angéline) (Fig. 8).

- Les assemblages 5 et 6 , à ammonites du Pliensbachien inférieur, surtout développés dans les Jebels Stah, Azreg, El Kohol, Bent Saïdane et Zaress (Fauré \& Peybernès 1986a, b; Dommergues et al. 1986; Rakús \& Guex 2002).

Seules les faunes du Sinémurien seront ici examinées (Fig. 8). L'analyse des assemblages fauniques du Pliensbachien inférieur sort du cadre que nous avons donné à ce travail.

\section{Assemblage 1 à Arnioceras $c f$. arnouldi}

C'est le niveau fossilifère le plus ancien du Sinémurien de Tunisie, identifié dans le seul Jebel Oust, où il a livré Arnioceras sp. ("niveau a» de Fauré \& Peybernès 1986a, b) et Arnioceras cf. arnouldi (Dumortier, 1867) («niveau 2» de Rakús \& Guex 2002), ainsi que des Phylloceratidae et des Lytoceratidae sans valeur chronostratigraphique. Ces auteurs se sont alors accordés sur un âge compatible avec la chronozone à Semicostatum nord-ouest européenne (voir aussi Guex et al. 2008).

Âge de l'assemblage 1. Dans le domaine téthysien, l'extension du genre Arnioceras est mal connue et les espèces qui lui sont rapportées sont peu informatives en termes biochronologiques. Sa première apparition est difficile à dater car dans de nombreuses régions de la marge nord-africaine (Haut-Atlas, Chaînes Bétiques), comme dans la Dorsale tunisienne, le genre apparait précocement dans la séquence fossilifere du Sinémurien, avant les premiers Asteroceratinae, et son âge ne peut être précisé (Braga et al. 1985; El Hariri et al. 1996, 2010; Lachkar et al. 1998). Pour ces premiers niveaux sans Asteroceras, un âge chronozone à Turneri à partie inférieure de chronozone à Obtusum serait admissible (Dommergues \& Meister 2017). À noter que dans le nord-ouest de l'Europe, $A$. arnouldi accompagne des Asteroceras tardifs de la sous-chronozone à Stellare (Dommergues 1993).

Assemblage 2 à Parasteroceras rakusi

Très fossilifère dans les carrières situées à l'E-S.E. du Jebel Oust (carrière Ayach Belkahla), ce second niveau nous a livré ( «niveau b» de Fauré \& Peybernès 1986a, b et Dommergues et al. 1986): Parasteroceras rakusi Dommergues, Fauré \& Peybernès, 1986, Arnioceras fieldingiceroides Dommergues, Fauré \& Peybernès, 1986, Oxynoticeroides simplicatum Dom- 
mergues, Fauré \& Peybernès, 1986, ? Oxynoticeroides sp. (in Dommergues \& Meister 2017: 259), Epophioceras aff. longicella (Quenstedt, 1885), Aegasteroceras? sp. et Microderoceras gr. nothum (Meneghini, 1898) - birchiades (Rosenberg, 1909). Rakús \& Guex (2002) y ajoutent, provenant de la «Carrière romaine» ("niveaux 4 et 5 » texte-fig. 3), Partschiceras tenuistriatum (Meneghini, 1868), Parasteroceras peyssonneli Rakús \& Guex, 2002 et Protocymbites azzouzi Rakús \& Guex, 2002.

Cette association est attribuée à la chronozone à Obtusum par Fauré \& Peybernès (1986a, b) et Dommergues et al. (1986). Pour Rakús \& Guex (2002) et Guex et al. (2008), elle serait plus ancienne et relèverait de la seule chronozone à Turneri.

Discussion. Bien que très riche et diversifiée, cette association est biostratigraphiquement peu contrainte car largement constituée de taxons originaux qui n'ont à ce jour jamais été formellement reconnus en dehors de la Tunisie, et réputés endémiques. Leur attribution stratigraphique est difficile à confronter au cadre chronostratigraphique donné par les ammonites dans la région téthysienne (Blau \& Meister 2000).

- Le genre Asteroceras y est absent, alors qu'il est pourtant très largement répandu sur la marge nord-africaine durant toute la chronozone à Obtusum. Il est très peu probable que des raisons paléoécologiques ou paléogéographiques ne lui aient pas permis de coloniser aussi l'Atlas tunisien, ce que confirme maintenant sa découverte dans le Jebel Ressas. Cette absence rend ainsi très peu probable l'attribution de l'assemblage 2 à la chronozone à Obtusum.

- Le genre Parasteroceras, avec la seule espèce $P$. rakusi, est un Asteroceratinae original, apparemment endémique au Sinémurien tunisien où il abonde, dont l'âge est difficile à replacer dans les échelles classiques. Les rares citations confirmées de ce genre en dehors de la Tunisie proviennent du Haut-Atlas (El Hariri et al. 1996) avec Parasteroceras cf. rakusi (= Parasteroceras sp. juv. sensu Dommergues \& Meister 2017). Ce taxon y est postérieur aux niveaux à Asteroceras gr. varians Fucini, 1903. Son âge ne peut ainsi être plus ancien que la partie moyenne à supérieure de la chronozone à Obtusum. Pour Dommergues \& Meister (2017: 247), il pourrait aussi, au moins partiellement, s'étendre sur la chronozone à Oxynotum.

- Le genre Aegasteroceras est présent dans ce niveau à de rares exemplaires (Fauré \& Peybernès 1986a, b) avec l'espèce "Parasteroceras" peyssonneli Rakús \& Guex, 2002, récemment recombinée dans le genre Aegasteroceras (Guex et al. 2008; Edmunds et al. 2016). Sa présence est un nouvel argument pour rajeunir ce niveau à la partie terminale de la sous-chronozone à Stellare, voire à la sous-chronozone à Denotatus.

Plusieurs Asteroceratinae voisins, à la morphologie capricorne de type Aegasteroceras plus ou moins prononcée, coexistent avec le genre Parasteroceras dans le Haut-Atlas marocain: par exemple "Asteroceras nov. sp.» in El Hariri et al. (1996: pl. 68, fig. 1-2) (= Asteroceras (?) sp. (A.) sensu Dommergues \& Meister 2017) et "Parasteroceras sp." in El Hariri et al. (1996: pl. 67, figs 19, 20) (= Parasteroceras (?) sp. (A.) sensu Dommergues \& Meister [2017]). Cette morphologie est aussi bien exprimée par plusieurs ammonites de la partie terminale de la chronozone à Obtusum du Jebel Bou Hamid (Haut-Atlas). Par exemple: «Asteroceras nov.sp.» in Lachkar et al. (1998: fig. 5.18-19) (= Asteroceras sp. (B) sensu Dommergues \& Meister [2017]); Aegasteroceras cf. blakei (Spath, 1925) sensu Guex et al. (2008) et $A$. aff. saggitarium (Blake, 1876) sensu Guex et al. (2008). Mais, dans le Haut-Atlas, ces couches ne renferment aucun spécimen attribuable au genre Parasteroceras.

- Le genre Oxynoticeroides y est représenté par plusieurs exemplaires de l'espèce unique $O$. simplicatum. Ce taxon hyperoxycône micro-ombiliqué, peu orné et à suture simplifiée, est placé lors de sa description originale (Dommergues et al. 1986) dans les Asteroceratinae, en raison de son âge, chronozone à Obtusum vraisemblable, qui le démarquait des autres taxons oxycônes du "Lotharingien " (Paroxynoticeras, Oxynoticeras) et du Pliensbachien (Radstockiceras, Carixiceras), avec lesquels il montre une importante convergence morphologique. Le genre est réattribué à la famille des Oxynoticeratidae par Rakús \& Guex (2002) et Dommergues \& Meister (2017). Sa mise en synonymie avec le genre Radstockiceras par Howarth (2013) ne peut être suivie. Ce taxon, possiblement endémique à la Tunisie, ne peut contribuer à préciser l'âge de l'assemblage 2, mais sa position, au sommet de la sous-chronozone à Stellare, sous-chronozone à Denotatus, voire, à la base de la chronozone à Oxynotum, est plus vraisemblable.

- Le genre Epophioceras, représenté par E. aff. longicella (Quenstedt, 1885), est le seul taxon que l'on puisse rapprocher des formes de la chronozone à Obtusum d'Europe du Nord-Ouest, bien que la forme tunisienne possède des traits originaux qui limitent cette assimilation. Contrairement à Rakús \& Guex (2002) nous l'avons trouvé en association, au Jebel Oust, avec les taxons de l'assemblage 2, et non dans un horizon postérieur. L'espèce nominale E. longicella serait présente en Angleterre dans la partie inférieure de la souschronozone à Stellare (Page 2003) et en Bourgogne, dans la partie supérieure à terminale de la sous-chronozone à Stellare (horizons à Confusum et à Blakei de Dommergues 1993).

- "Protocymbites azzouzi» Rakús \& Guex, 2002 est une forme micromorphe connue à ce jour par un seul spécimen, provenant du Jebel Oust.

Âge de l'assemblage 2. L'absence du genre Asteroceras et la présence du genre Aegasteroceras concourrent à le situer dans une tranche de temps étendue de la partie terminale de la souschronozone à Stellare, à un niveau équivalent du biohorizon à Sagittarium nord-ouest européen, à tout ou partie de la souschronozone à Denotatus (Fig. 8). L'absence d'Eparietites ne peut permettre d'écarter cet âge, car ce genre est totalement absent sur la marge sud-téthysienne. Son maintien «dans la zone à Turneri ou dans un niveau inférieur de la zone à Obtusum» (Rakús \& Guex 2002; Guex et al. 2008) ne peut pas être soutenu et son important rajeunissement s'accorde avec les hypothèses déjà avancées dans le Haut-Atlas marocain par El Hariri et al. (1996) et Wilmsen et al. (2002).

Assemblage 3 à Gleviceras gr. richei et Paroxynoticeras gr. salisburgense

Très bien documenté par Rakús \& Biely (1970) et Rakús \& Guex (2002), ces derniers auteurs y ont distingué deux niveaux sur la coupe du Jebel Oust: 


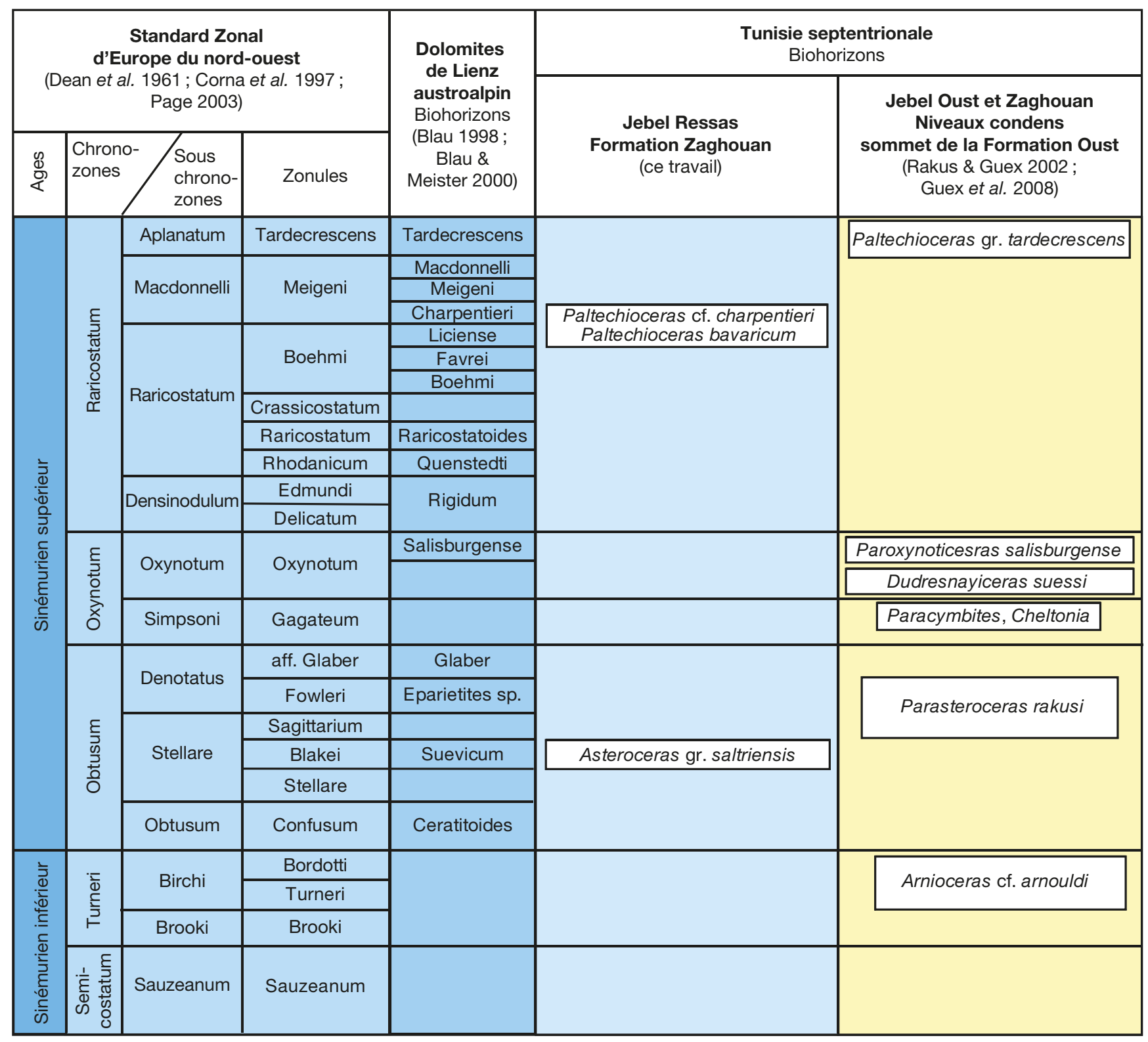

FIG. 8. - Succession des biohorizons d'ammonites du Sinémurien supérieur des deux aires paléogéographiques de la Tunisie septentrionale : Sillon tunisien, au sein de la Fm Zaghouan, ce travail; Dorsale tunisienne centrale, dans les Niveaux condensés du sommet de la Fm Oust, réinterprété de Rakús \& Guex (2002) et Guex et al. (2008). Comparaison avec le standard zonal établi en Europe du Nord-Ouest et avec la biozonation établie en domaine nord-téthysien par Blau \& Meister (2000).

- un «niveau à simplicatum-suessi» avec Juraphyllites planispiroides Rakús, 1994, Angulaticeras cf. densilobatum (Pompeckj, 1893), Gleviceras gr. richei (Rakús, 1994), Plesechioceras gr. delicatum (Buckman, 1914), P. aff. edmundi (Dumortier, 1867), Cheltonia aff. galatea (Hoffmann, 1944), Dudresnayiceras gr. suessi (Hauer, 1854)-suessiforme Rakús, 1994;

- un «niveau à salisburgense-birchiades» à Paroxynoticeras gr. salisburgense (Hauer, 1856)-aff. subundulatum (Pia, 1914), Microderoceras gr. birchiades Rosenberg, 1909-nothum (Meneghini, 1898).

- Nous y ajoutons, à la partie inférieure, le «niveau à Paracymbites» que Guex et al. (2008) situaient au sommet de la chronozone à Obtusum et qui renferme Paracymbites dennyiformis Rakús \& Guex, 2002, "Protocymbites» oustense (Rakús \& Guex, 2002) et Bouhamidoceras zizense Dubar, 1961.
Âge de l'assemblage 3. À l'exception de rares espèces micromorphes, toutes sont des espèces téthysiennes connues à un niveau identique sur la totalité de la marge maghrébine de la Téthys (Lachkar et al. 1998; Guex et al. 2008; El Hariri et al. 2010). Elles sont parfaitement représentatives de la chronozone à Oxynotum, vraisemblablement de sa partie supérieure (horizon à Salisburgense de Blau \& Meister 2000) (Fig. 8).

\section{Assemblage 4 à Paltechioceras gr. tardecrescens}

Uniquement identifié sur la coupe Attaque Angeline, entre Jebel Stah et Grand Zaghouan (Rakús \& Biely 1970), c'est le «niveau 6» de Rakús \& Guex (2002) qui a fourni à ces auteurs des Echioceratidae évocateurs de la chronozone à Raricostatum, sans plus de précision ("zone à Hierlatzicum- 
Rothpletzi » sensu Rakús \& Guex 2002). Tous appartiennent au même type, évolute, à costulation assez forte et rigide, qui les placent dans le groupe de Paltechioceras tardecrescens (Hauer, 1856). Ce taxon est bien représenté sur la marge maghrébine de la Téthys (El Hariri et al. 1996, 2010; Wilmsen et al. 2002; Guex et al. 2008) où partout, comme en Tunisie, il termine la séquence fossilifère du Sinémurien à un niveau équivalent à la sous-chronozone à Aplanatum nord-ouest européenne (Fig. 8).

\section{Assemblages 5 et 6}

Ces derniers niveaux condensés relèvent du Pliensbachien inférieur, "zone à Aenigmaticum (assemblage 5) et zone à Demonense» (assemblage 6) sensu Rakús \& Guex (2002). Le dernier hard-ground clôture la Fm Oust. Ils sont particulièrement documentés et illustrés d'après des récoltes des mines de fluorine du Jebel Stah (Rakús \& Guex 2002; Dommergues et al. 2004). Cette même localité nous a livré (Fauré \& Peybernès 1986a, b) des ammonites représentatives de ces deux biozones avec: Tropidoceras flandrini (Dumortier, 1869), T. demonense (Gemmellaro, 1884), T. mediterraneum (Gemmellaro, 1884), T. zitteli Fucini, 1899, Miltoceras sella (Gemmellaro, 1884), Metaderoceras gr. evolutum (Fucini, 1924)-gemmellaroi (Levi, 1896), Radstockiceras gemmellaroi (Pompeckj, 1907) et Galaticeras gr. aegoceroides (Gemmellaro, 1884).

\section{LE CADRE CHRONOSTRATIGRAPHIQUE}

La biozonation proposée ici, pour le Sinémurien de la Tunisie, intègre l'ensemble des données stratigraphiques offertes par les «Niveaux condensés», que nous réinterprétons ici, et les données acquises par le présent travail dans la Fm Zaghouan du Jebel Ressas, en domaine de sédimentation non condensée.

\section{SINÉMURIEN INFÉRIEUR (PARTIM) \\ Chronozone à Turneri}

Sa présence ne peut être établie sans ambiguïté en l'absence du genre Caenisites en Tunisie. La chronozone est possiblement représentée par le niveau à Arnioceras seuls, dont Arnioceras cf. arnouldi, avec lequel commence la séquence fossilifère du Sinémurien tunisien dans le Jebel Oust (Rakús \& Biely 1970 ; Fauré \& Peybernès 1986a, b; Rakús \& Guex 2002).

Un même âge, chronozone à Turneri, est admis pour les horizons à Arnioceras seul, du groupe de $A$. rejectum Fucini, qui précèdent l'apparition du genre Asteroceras dans le Haut-Atlas marocain (El Hariri et al. 1996, 2010; Lachkar et al. 1998), ainsi que dans les Cordillières Bétiques (Braga et al. 1985).

\section{SINÉMURIEN SUPÉRIEUR}

Chronozone à Obtusum

Sous-chronozone à Stellare. Nous lui attribuons l'association Asteroceras gr. saltriensis, A. cf. meridionalis, Arnioceras gr. ceratiticum-rejectum et Epophioceras aff. landrioti, en l'absence du genre Parasteroceras. Les Asteroceras sont des formes tardives du genre caractérisant la partie supérieure de la sous-chronozone à Stellare et leur âge est vraisemblablement proche de celui de l'horizon à Suevicum de l'Austroalpin supérieur (Blau \& Meister 2000) ou de l'horizon à Blakei nord-ouest européen (Dommergues 1993; Page 2003).

La sous-chronozone est nouvellement identifiée dans le Jebel Ressas, dans un contexte sédimentaire non ou peu condensé. Curieusement, elle ne semble représentée dans aucun des "Niveaux condensés» étudiés, à ce jour, dans la Dorsale tunisienne.

Sous-chronozone à Stellare terminale à sous-chronozone à Denotatus. Le niveau que nous attribuons ici à cet intervalle renferme Parasteroceras rakusi, Oxynoticeroides simplicatum, Arnioceras fieldingiceroides, Epophioceras aff. longicella, Aegasteroceras peyssonnelli et "Protocymbites" azzouzi.

Nous avons discuté plus haut en détail des raisons qui justifiaient son attribution à cet intervalle par l'absence du genre Asteroceras, alors que sont présents de rares représentants du genre Aegasteroceras.

Cet âge permet d'éclairer d'un jour nouveau la position du genre Parasteroceras, en particulier du taxon P. rakusi, dans le schéma évolutif des Asteroceratinae, en le plaçant à un stade tardif de l'évolution de la sous-famille, assurant, dans la souschronozone à Denotatus, la transition entre les genres Asteroceras et Paroxynoticeras, genre qui se développe ensuite dans la chronozone à Oxynotum (Dommergues \& Meister 2017).

Cette association est largement représentée dans les Niveaux condensés du sommet de la Fm Oust des seuls Jebels Oust et Zaghouan central (Fauré \& Peybernès 1986a, b; Rakús \& Guex 2002). Elle n'est pas identifiée dans les calcaires pélagiques de la Fm Zaghouan.

\section{Chronozone à Oxynotum}

Les associations sont celles énumérées dans l'assemblage 3 des «Niveaux condensés" avec, pour les principales espèces, Bouhamidoceras zizense, Gleviceras gr. richei, Paroxynoticeras aff. salisburgense, Plesechioceras gr. delicatum, Microderoceras gr. birchiades, Angulaticeras cf. densilobatum, Protocymbites oustense et Paracymbites dennyiformis. Leur âge est plutôt évocateur de la partie supérieure de la chronozone, mais le contexte de condensation ne permet pas de préciser quelles seraient les équivalences des trois horizons de Rakús \& Guex (2002) au sein de la sous-chronozone à Oxynotum nord-ouest européenne.

\section{Chronozone à Raricostatum}

Des Echioceratidae du genre Paltechioceras permettent d'identifier la chronozone sans ambiguïté. Par contre, l'absence de succession stratigraphique claire et reproductible des espèces rend sa subdivision difficile. Il apparaît toutefois clairement que les populations d'Echioceratidae de la Fm Zaghouan du Ressas et les populations figurées par Rakús \& Guex (2002) dans les Jebels Stah et Grand Zaghouan, ne sont pas identiques et qu'elles se succèdent dans le temps.

Sous-chronozone à Raricostatum. Cette population, identifiée à ce jour dans la seule Fm Zaghouan du Jebel Ressas, associe, 
à un niveau sensiblement identique, Paltechioceras bavaricum, $P$. cf. charpentieri et Juraphyllites aff. nardii, autant d'espèces absentes des Niveaux condensés du sommet de la Fm Oust.

Sur la marge nord de la Téthys, où ils se mêlent à quelques Echioceratidae nord-ouest européens, les Paltechioceras du groupe de P. bavaricum et $P$. rothpletzi se placent dans la partie sommitale de la sous-chronozone à Raricostatum (horizon à Liciense et Rothpletzi de Blau \& Meister 2000). Dans cette même région (Autroalpin supérieur autrichien), P. charpentieri est à peine plus récent puisqu'il caractérise plutôt la base de la sous-chronozone à Macdonnelli (horizon à Meigeni et Charpentieri de Blau \& Meister 2000).

Sous-chronozone à Aplanatum. Tous les Paltechioceras figurés par Rakús \& Guex (2002) dans le dernier des Niveaux condensés sinémuriens du sommet de la Fm Oust appartiennent au groupe de P. tardecrescens (Hauer, 1856). Il s'agit d'un Paltechioceras tardif, largement répandu dans la Téthys méditerranéenne où il marque toujours le sommet de la séquence sinémurienne (horizon à Tardecrescens et Romanicum de Blau \& Meister 2000), mais également sur les plates-formes nord-ouest européennes où il est représentatif de la partie sommitale de la sous-chronozone à Aplanatum. Aucune de ces formes n'a été identifiée en Tunisie, dans un contexte non condensé.

\section{SIGNIFICATION SÉDIMENTOLOGIQUE}

Il est singulier de constater que les associations d'ammonites enregistrées dans les domaines pélagiques et condensés ne sont pas identiques mais se complètent au contraire, avec respectivement (Fig. 8):

- En domaine condensé: chronozone à Turneri; souschronozone à Stellare terminale à sous-chronozone à Denotatus; chronozone à Oxynotum; sous-chronozone à Aplanatum.

- En domaine pélagique: sous-chronozone à Stellare; souschronozones à Raricostatum et à Macdonnelli.

Qu'elles aient été prélevées en domaine pélagique ou en domaine condensé, les associations d'ammonites qui se succèdent témoignent de périodes d'enregistrement très discontinues, brèves, dont la durée est de l'ordre de celle d'un horizon d'ammonites, toujours très inférieure à celle d'une chronozone ou d'une sous-chronozone (Fig. 8). L'enregistrement est à la fois déterminé par les fluctuations relatives du niveau marin et les conditions paléogéographiques et sédimentologiques locales, souvent complexes, qui règnent sur la marge nord-africaine, à l'articulation entre plate-forme carbonatée méridionale et bassins pélagiques atlasiques.

\section{PALÉOBIOGÉOGRAPHIE}

Les faunes d'ammonites du Sinémurien supérieur tunisien sont majoritairement représentées par des taxons totalement endémiques aux régions téthysiennes déjà observés à un niveau stratigraphique identique dans d'autres secteurs de la Téthys méditerranéenne comme l'Austroalpin supérieur (Blau 1998;
Blau \& Meister 2000), les Monts du Bakony (Géczy \& Meister 2007), les Apennins (Cecca et al. 1987; Dommergues et al. 1994) et le Haut-Atlas marocain (El Hariri et al. 1996, 2010; Lachkar et al. 1998; Guex et al. 2008) (Fig. 5).

Le particularisme exprimé par les faunes du Sinémurien supérieur tunisien, déjà souligné par Dommergues et al. (1986), puis bien documenté par Rakús \& Guex (2002), repose sur l'existence dans la Dorsale tunisienne d'un certain nombre de taxons originaux qui se sont avérés, encore à ce jour, totalement inconnus à l'extérieur de cette région. Les causes de cet endémisme localisé des ammonites ont été discutées, notamment, par Dommergues \& El Hariri (2002) et Dommergues et al. (2004).

C'est dans la chronozone à Obtusum que les travaux de Rakús \& Guex (2002) font apparaître le particularisme le plus marqué avec les taxons suivants, Parasteroceras rakusi, Oxynoticeroides simplicatum, Aegasteroceras peyssonneli, «Protocymbites" azzouzi, connus de la seule Dorsale tunisienne, et jamais décrit ailleurs.

L'absence d'Asteroceras dans ces associations pouvait paraitre singulière et évoquer l'existence d'un particularisme paléobiogéographique local, car ce genre est par ailleurs très répandu dans toutes les autres régions de la Téthys méditerranéenne, ainsi que sur les plates-formes européennes. La découverte d'ammonites de ce genre (Asteroceras gr. saltriensis et $A$. cf. meridionalis) dans la Fm Zaghouan du Jebel Ressas permet donc de relativiser l'isolement géographique de la Dorsale tunisienne durant la chronozone à Obtusum. Une même remarque peut être faite pour Arnioceras gr. ceratitoides-rejectum, jusque-là non identifié en Tunisie, qui est pourtant très largement répandu, notamment sur la marge nord-africaine, jusqu'au Haut-Atlas.

Avec la chronozone à Oxynotum tout particularisme tunisien disparaît totalement et, à l'exception d'éléments micromorphes connus à de rares exemplaires, souvent uniques (Paracymbites dennyiformis, Protocymbites oustense), tous les taxons cités dans cette chronozone par Rakús \& Guex (2002) sont les formes connues dans de nombreuses régions de la Téthys méditerranéenne, en particulier au Maroc (Juraphyllites planispiroides, Bouhamidoceras zizense, Paroxynoticeras gr. salisburgense-subundulatum, Gleviceras gr. richei, Dudresnayiceras gr. suessi-suessiforme, Angulaticeras cf. densilobatum, Microderoceras gr. birchiades-nothum), voire sur les plates-formes ouesteuropéennes (Plesechioceras gr. delicatum-edmundi).

Dans la chronozone à Raricostatum, Juraphyllites aff. nardii et les Paltechioceras bavaricum et $P$. cf. charpentieri sont encore des taxons méditerranéens, connus dans d'autres régions téthysiennes comme l'Austroalpin, les Apennins et le Haut-Atlas, les deux dernières étant également fréquemment signalées dans la partie méridionale du craton européen (Préalpes, Hug 1899; Pyrénées, Fauré \& Alméras 2006). Paltechioceras tardecrescens, qui clôture la séquence du Sinémurien de Tunisie, est le seul taxon que l'on puisse considérer comme ubiquiste car largement représenté dans toute la Téthys méditerranéenne (Blau 1998; Lachkar et al. 1998; El Hariri et al. 2010) ainsi que sur les plates-formes ouest européennes (Getty 1973; Dommergues 1993), mais également le Domaine pacifique (Smith 1981). 


\section{CONCLUSIONS}

\section{CONCLUSIONS À LA RÉVISION STRATIGRAPHIQUE DU JEBEL RESSAS}

Nous retrouvons dans le Jebel Ressas la même succession lithostratigraphique que dans la partie septentrionale de la Dorsale tunisienne et c'est avec les faciès du Jurassique du Jebel Bou Kornine d'Hammam-Lif que les analogies sont les plus frappantes. Comme dans ce jebel, une puissante assise calcaréo-dolomitique correspondant à la Fm Oust (Trias supérieur à Sinémurien inférieur) est surmontée de calcaires à silex correspondant à la Fm Zaghouan. Dans cette formation sont pour la première fois reconnues les chronozones à Obtusum et à Raricostatum du Sinémurien supérieur. Ces datations s'ajoutent aux datations au Pliensbachien (chronozone à Ibex) établies dans la partie supérieure de cette même formation dans les Jebels Zaghouan et Bou Kornine de Hammam-Lif (Fauré et al. 2007; Sekatni et al. 2013) et confirment l'âge Sinémurien supérieur-Pliensbachien inférieur de la Fm Zaghouan de Tunisie.

Les Fm Bou Gabrine (Pliensbachien pars) et Fm Stah (Toarcien pars) y sont présentes et bien datées. Avec les calcaires à zoophycoos de la Fm Kef el Orma (Toarcien supérieur à Bajocien inférieur pars) apparaissent, en même temps que dans le Jebel Bou Kornine, les premiers épandages conglomératiques et calciturbiditiques témoignant de premiers mouvements extensifs qui affectent la marge nord-africaine (Sekatni et al., 2008). Sous un faciès "ammonitico rosso" identique à celui des autres jebels de la Dorsale tunisienne, la Fm Zaress est réduite au seul Oxfordien supérieur-Kimméridgien inférieur, alors que ses dépôts s'échelonnent par ailleurs (Zaghouan, Bou Kornine) du Callovien moyen (chronozone à Anceps) au Kimméridgien inférieur (Sekatni-Aich 2010 ; Sekatni et al. 2013).

Comme dans le Jebel Bou Kornine d'Hammam-Lif (SekatniAich 2010), une importante discontinuité sépare les Fm Kef el Orma et Zaress. Dans le Jebel Ressas, elle matérialise la lacune de dépôts échelonnés du Bajocien inférieur pars à l'Oxfordien supérieur. Cette période correspond à la totalité de la Fm Bent Saïdane, dont de rares témoins, présentant des faciès pseudoconglomératiques et bréchiques identiques à celui des "Conglomérats supérieurs" du Jebel Bou Kornine sont conservés dans le nord du Jebel Ressas. La quasi-totale absence de la Fm Bent Saïdane et de la partie inférieure de la Fm Zaress pourrait ici s'expliquer par un glissement synsédimentaire en masse sur une paléopente s'inscrivant dans un dispositif en blocs basculés de type marge passive en extension (Sekatni et al. 2013).

\section{Conclusion À L'ÉTUde du SinÉMURIEn de Tunisie}

Ce travail confirme l'exceptionnelle diversité de la faune d'ammonite du Sinémurien de la Tunisie septentrionale. À la trentaine de taxons décrits et figurés par Rakús \& Guex (2002) dans les Niveaux condensés du sommet de la Formation Oust de la Dorsale tunisienne, nous ajoutons plusieurs genres et espèces nouveaux pour la Tunisie, découverts dans les calcaires pélagiques non condensés attribués à la Formation Zaghouan, dans le Jebel Ressas.
Ces ammonites permettent d'identifier deux horizons stratigraphiques qui n'avaient pas été, à ce jour, identifiés dans les niveaux condensés, dont la faune est pourtant particulièrement riche et diversifiée (Rakús \& Guex 2002). Elles permettent notamment de bien identifier la chronozone à Obtusum, avec des ammonites de la partie supérieure de la sous-chronozone à Stellare (Asteroceras gr. saltriensis, A. cf. meridionalis, Arnioceras gr. ceratitoides-rejectum, Epophioceras aff. landrioti), puis la partie moyenne de la chronozone à Raricostatum, avec plusieurs espèces de Paltechioceras, également non signalées en Tunisie à ce jour (P. bavaricum, $P$. cf. charpentieri).

Ces nouveaux repères stratigraphiques s'insèrent parfaitement dans le canevas biochronologique déjà établi dans la Dorsale tunisienne par l'étude des seuls Niveaux condensés du sommet de la Fm Oust (Rakús \& Guex 2002; Guex et al. 2008) et le complètent. Ils nous permettent une nouvelle approche en nous amenant à amender l'âge des associations d'ammonites contemporaines de l' "horizon à Parasteroceras rakusi», attribué par ces auteurs à la chronozone à Turneri, et à les placer dans une tranche de temps pouvant s'étendre de la partie tout à fait terminale de la sous-chronozone à Stellare à tout ou partie de la sous-chronozone à Denotatus (chronozone à Obtusum).

Bien que de nombreux taxons du Sinémurien supérieur décrits par Rakús \& Guex (2002) n'aient, à ce jour, pas été signalés en dehors de la Tunisie, cette étude nous amène à relativiser la réalité d'un endémisme tunisien au Sinémurien. En effet, les faunes nouvellement décrites dans le présent article sont toutes des formes téthysiennes "classiques» déjà observées à un niveau stratigraphique identique dans d'autres régions de la Téthys méditerranéenne (e.g. Austroalpin, Montagne de Bakony, Apennins, Haut-Atlas). Il en est de même pour la quasi-totalité des espèces énumérées par Rakús \& Guex (2002) dans les chronozones à Oxynotum et à Raricostatum de la Dorsale tunisienne qui toutes se retrouvent, à l'identique, notamment, dans le Haut-Atlas (El Hariri et al. 1996, 2010; Lachkar et al. 1998; Guex et al. 2008). Seul le «niveau à Parasteroceras rakusi» de la dorsale, et les taxons d'ammonites qui lui sont subordonnés, pourrait apparemment constituer une exception si la réalité de leur absence en dehors de la Tunisie était avérée. Les couches correspondant à l'équivalent du sommet de la sous-chronozone à Stellare et à la chronozone à Denotatus, qui pourraient les renfermer restent en effet insuffisamment identifiées sur la marge nord-africaine et leur faune encore non décrite.

\section{Remerciements}

Nos remerciements les plus chaleureux s'adressent à $M$. le Professeur F. Zargouni qui a suggéré et supervisé cette étude en mettant tous les moyens de l'université de Tunis à notre service.

Les ammonites de l'Oxfordien et du Kimméridgien inférieur du Jebel Ressas ont été déterminées par le Professeur Ch. Mangold dont nous saluons ici la mémoire.

P. Culleton a supervisé la traduction du résumé anglais.

Nous tenons à remercier les rapporteurs P. Neige (Université de Bourgogne) et C. Meister (Muséum d'Histoire naturelle de Genève) pour leurs remarques et leur conseils pertinents qui ont contribué à l'amélioration du manuscrit. 


\section{RÉFÉRENCES}

Alkaya F. \& Meister C. 1995. - Liassic ammonites from the Central and Eastern Pontides (Ankara and Lelkit area, Turkey). Revue de Paléobiologie 14 (1): 125-193.

ARKELL W. J. 1951. - Proposed use of the plenary powers to designate the type species of the genus "Arnioceras" Hyatt, 1867 (Class Cephalopoda, Order Ammonoidea). Bulletin of the Zoological Nomenclature 2: 217-219. https://www.biodiversitylibrary.org/ page/12020594

BALTZER A. VON 1895. - Versteinerungen aus dem tunisischen Atlas. Neues Jahrbuch für Mineralogie, Geologie und Palaeontologie 1: 105-107.

Bismuth H., Bonnefous J. \& Dufaure Ph. 1967. - Mesozoic microfaciès of Tunisia. Guide book to the Geology and History of Tunisia. Petroleum Exploration Society of Lybia, Amsterdam: 159-173.

BLAU J. 1998. - Monographie der Ammoniten des Obersinemurium (Lotharingium, Lias) der Lienzer Dolomiten (Österreich): Biostratigraphie, Systematik and Paläobiogeographie. Revue de Paléobiologie 17 (1): 177-285.

Blau J. \& Meister C. 2000. - Upper Sinemurian Ammonites Successions Based on 41 Faunal Horizons : an Attempt at Worldwide Correlation. GeoResearch Forum 6: 3-12.

Blau J., Meister C., Schlatter R., Schmidt-Effing R. 2003.Ammonites from the Lower Jurassic (Sinemurian) of Tenango de Doria (Sierra Madre Oriental, Mexico). Part III: Echioceratidae: Revue de Paléobiologie 22 (1): 421-437.

BONNEFOUS J. 1972. - Contribution à l'étude stratigraphique et micropaléontologique du Jurassique de la Tunisie (Tunisie septentrionale et centrale, Sahel, zone des Chotts). Thèse Paris IV, t. 1-3, 397 p.

Bonnefous J. \& RaKús M. 1965. - Précisions nouvelles sur le Jurassique du Jebel Bou Kornine d'Hammam-Lif (Tunisie). Bulletin de la Société géologique de France 6 (7): 855-858. https://doi. org/10.2113/gssgfbull.S7-VII.6.855

BÖSE E. 1894. - Über liasische und mittel jurassische Fleckenmergel in den bayerischen Alpen. Zeitschrift der Deutschen geologischen Gesellschaft 46: 703-768.

Braga J.-C., Martin-Algarra A. \& Rivas P. 1985. — Ammonites du Lias inférieur (Sinémurien-Lotharingien) de la Sierra Harana (Cordillères bétiques, Espagne), in Premier Colloque du Centre International d'Etude du Lias (CIEL). Cahiers de l'Université Catholique de Lyon 14: 85-100.

Braga J.-C., Comas-Rengifo M. J., Goy A. \& Rivas P. 1982. Comparaciones faunisticas y correlaciones en el Pliensbachiense de la Zona Subbética y Cordillera Ibérica. Boletim de la Real Societad Espanola de Historia Natural (Geologia) 80: 221-244.

BucKMan S. S. 1909-1930. — Yorkshire Type Ammonites. Welseyand Son, Londres, vol. I-II: i-xvi, 1-121, pl. 1-130; suivi de Type Ammonites. Weldon and Welsey, Londres, vol. III-VII. https:// doi.org/10.5962/bhl.title.45669

Bujalka P., Johan K., Krivy M., Rakús M. \& VaceK J. 1971. Carte géologique de la Tunisie à 1/50000. Feuille no 29, Grombalia. Carte et notice explicative, $93 \mathrm{p}$.

CASSINIS G. \& CANTAluPPI G. 1967. - Nuovi dati paleontologici per una più approfondita conoscenza del limite cronologico superiore della "Corna» di Botticino (Brescia). Atti dell'istituto geologico della università di Pavia XVIII: 51-64.

CANTALUPPI G. \& CORTI S. 1969. — La fauna della «piedra di Moltrasio" (Como). Società Italiana di Scienza Naturali e del Museo civico di Storia naturale di Milano 60 (2): 103-133.

CASTANY G. 1951. - Étude géologique de l'Atlas tunisien. Royaume de Tunis. Ministère des travaux publics, service des mines, de l'industrie et de l'énergie. Impr. "la Rapide", Tunis, 632 p. (Annales des Mines et Géologie; 8).

CASTANY G. 1955. - Les extrusions jurassiques en Tunisie. Royaume de Tunis. Ministère des travaux publics, service des mines, de l'industrie et de l'énergie. Impr. "la Rapide", Tunis, 71 p. (Annales des Mines et Géologie; 14).
Cecca F., Dommergues J.-L., Mouterde R. \& Pallini G. 1987. - Ammonites méditerranéennes du Lotharingien de Gorgo a Cerbara (Monte Nerone, Apennin des Marches, Italie), in Deuxième Colloque du Centre international d'Étude du Lias (CIEL). Cahiers de l'Institut catholique de Lyon, Série Sciences 1: $67-82$

CORNA M. 1987. — Les horizons sinémuriens du Calcaire à gryphées du Jura méridional français (zone à Conybeari-zone à Obtusum). Geobios 20 (4): 531-536. https://doi.org/10.1016/ S0016-6995(87)80085-2

Corna M., Dommergues J.-L., Meister C. \& Mouterde R. 1997. - Sinémurien, in CARIOU E. \& HANTZPERGUE P. (coords), Biostratigraphie du Jurassique ouest-européen et méditerranéen: zonations parallèles et distribution des invertébrés et microfossiles. Groupe français d'étude du Jurassique. Bulletin des Centres de Recherche Exploration-Production Elf-Aquitaine, Mémoire 17: 9-14.

Corna M., Dommergues J.-L., Meister C. \& Page K. 1997. Les faunes d'ammonites du Jurassique inférieur (Hettangien, Sinémurien et Pliensbachien) au nord du massif des Écrins (Oisans, Alpes occidentales françaises). Revue de Paléobiologie 16 (2): 321-409.

Dean W. T., Donovan D. T. \& Howarth M. K. 1961. - The Liassic Ammonite zones and subzones of the North West European province. Bulletin of the British Museum (Natural History) Geology 4: 435-505. https://www.biodiversitylibrary.org/page/36359242

Dommergues J.-L. 1993. — Les ammonites du Sinémurien supérieur de Bourgogne (France) : biostratigraphie et remarques paléontologiques. Revue de Paléobiologie 12 (1): 67-173.

Dommergues J.-L. \& El Hariri K. 2002. - Endemism as a palaeobiogeographic parameter of basin history illustrated by early- and mid-Liassic peri-Tethyan ammonites fauna. Palaeogeography, Palaeoclimatology, Paleoecology 184: 407-418. https:// doi.org/10.1016/S0031-0182(02)00276-6

Dommergues J.-L. \& Meister C. 2017. — Ammonites du Jurassique inférieur (Hettangien, Sinémurien, Pliensbachien) d'Afrique du Nord (Algérie, Maroc, Tunisie). Atlas d'identification des espèces. Revue de Paléobiologie 26 (2): 189-367.

Dommergues J.-L., Fauré Ph. \& Peybernès B. 1986. - Le Lotharingien inférieur du Jebel Oust; description d'ammonites nouvelles (Asteroceratinae, Arieticeratinae). Comptes-rendus de l'Académie des Sciences de Paris 302: 1111-1116. https://gallica. bnf.fr/ark:/12148/bpt6k5675350v/f1117.item

Dommergues J.-L., C. Meister \& Mettraux M. 1990. — Succession des faunes d'ammonites du Sinémurien et du Pliensbachien dans les Préalpes romandes (Vaud et Fribourg). Geobios 23 (3): 307-341. https://doi.org/10.1016/0016-6995(90)80004-Y

Dommergues J.-L., Ferreti A. \& Meister C. 1994. — Les faunes du Sinémurien de l'Apennin Central (Marches et Toscane, Italie). Bolletino della Società Paleontologica Italiana 33 (1): 13-42.

Dommergues J.-L., Meister C. \& BöHM F. 1995. - New data on Austroalpine Liassic Ammonites from the Adnet quarries and adjacent areas (berösterreich, Northern Calcareous Alps). Jahrbuch des Geologischen Bundesanstalt 138: 161-205.

Dommergues J.-L., Meister C., Soussi F. \& Abdallah H. 2004. - Paratropidoceras nov. gen. numidianum nov. sp. et Tunisiceras nov. gen. insolitus nov. sp., deux nouveaux genres et de deux nouvelles espèces d'ammonites du Carixien de la Téthys méditerranéenne (Pliensbachien, Tunisie). Revue de Paléobiologie 23 (2): 463-475.

Dommergues J.-L., Forest-Bize N., Gely J. P. \& Loreay J. P. 2005. - Les faunes d'ammonites du Sinémurien supérieur (Jurassique inférieur) du Perron des Encombres (Alpes occidentales, Zone subbriançonnaise entre Arc et Isère). Revue de Paléobiologie 24 (2): 673-696.

Dommergues J.-L., CATANeO G., AÏTE R. \& GÉlard J.-P. 2008. Les ammonites de l'Hettangien, du Sinémurien et du Pliensbachien inférieur de la Dorsale de Grande Kabylie (Algérie). Geodiversitas 30: 539-576. 
Donovan D. T. 1958. - The Lower Liassic Ammonite fauna from the Fossil Bed at Langeneckgrat, near Thun (Median Alps). Mémoires suisses de Paléontologie 74: 1-58.

Dubar G. 1953. - Gisements liasiques de la Dorsale tunisienne (Djebel Bent-Saïdane et Dj. Zaghouan). Comptes-Rendus sommaires de la Société géologique de France: 354-356.

Edmunds M., Whicher J., Langham P. \& Chandler R. B. 2016. - A systematic account of the ammonite fauna of the Obtusum Zone (Sinemurian Stage, Lower Jurassic) from Marston Magna, Somerset, UK. Proceedings of the Geologists' Association 127 (2): 146-171.

El Hariri K., Dommergues J.-L., Meister C. \& Chafiki D. 2010. - Nouvelles données sur les ammonites du Sinémurien et du Pliensbachien basal du Haut-Atlas central (Maroc). Taxonomie et implication stratigraphiques et paléobiogéographiques. Revue de Paléobiologie 29: 217-260.

El Hariri K., Dommergues J.-L., Meister C., Souhel A. \& CHAFIKI D. 1996. - Les ammonites du Lias inférieur et moyen du Haut-Atlas central de Béni Mellal (Maroc) : taxinomie et biostratigraphie à haute résolution. Geobios 29: 537-576. https:// doi.org/10.1016/S0016-6995(96)80025-8

ELMI S. 2002. — L'Afrique du Nord au Jurassique, in International workshop sur le Jurassique de la marge Maghrébine de la Téthys. ATEIG-GFEJ, Tunis: 266-274.

Fauré Ph. 2002. - Le Lias des Pyrénées. Thèse Université Toulouse III. Strata, sér. II, 39: 1-761.

FAURÉ PH. \& AlMÉRAS Y. 2006. — Le Sinémurien terminal (zone à Raricostatum) des Pyrénées et des chaînons du Bas-Languedoc (France) : Biostratigraphie, évolution sédimentaire et paléogéographie. Bulletin de la Société d'Histoire naturelle de Toulouse 142: 5-25.

FaURÉ Ph. \& PeYBernès B. 1986a. - Biozonation par ammonites et essai de corrélation des séries réduites liasiques de la «Dorsale tunisienne», in 5e Conférence Internationale du P.I.G.C. (UNESCO) n 183, Marrakech. Revue de la Faculté des Sciences de Marrakech, Section Sciences de la Terre: 259-279.

Fauré Ph. \& Peybernès B. 1986b. - Biozonation par ammonites et essai de corrélation des séries réduites liasiques de la «Dorsale tunisienne». Bulletin de la Société d'Histoire naturelle de Toulouse 122: 41-49. https://gallica.bnf.fr/ark:/12148/bpt6k65562329/ f43.item

Fauré Ph., Alméras Y., Sekatni N. \& Zargouni F. 2007. — Le Pliensbachien du Jebel Zaghouan (Tunisie). Nouvelles données fauniques. Implications biostratigraphiques et paléobiogéographiques. Geodiversitas 29: 473-506.

Fauré Ph., SeKatni N. \& Arfaoui S. (sous presse). - Précisions stratigraphiques sur le Jurassique inférieur et moyen des Jebels Châabane et Djedidi (Tunisie). Paléontologie des ammonites, implications paléobiogéographiques. Bulletin de la Société d'Histoire naturelle de Toulouse.

FuCINI A. 1901. - Cefalopodi Liassici del Monte di Cetona. Palaeontographia Italica 7: 1-89.

FuCINI A. 1902. - Cefalopodi Liassici del Monte di Cetona. Palaeontographia Italica 8: 91-177.

FuCini A. 1903. - Cefalopodi Liassici del Monte di Cetona. Palaeontographia Italica 9: 125-185.

GéCZy B. \& Meister C. 2007. — Les ammonites du Sinémurien et du Pliensbachien inférieur de la Montagne du Bakony (Hongrie). Revue de Paléobiologie 26 (1): 137-305.

GETTY T. A. 1973. - A revision of the generic classification of the family Echioceratidae (Cephalopoda, Ammonoidea) (Lower Jurassic). The University of Kansas. Paleontological Contributions 64: 1-32 p.

Guérin-Franiatte S. 1966. - Ammonites du lias inférieur de France. Psilocerataceae: Arietitidae. Éditions du Centre National de la Recherche Scientifique (CNRS), Paris. 455 p.

Guex J., Rakús M., Morard A. \& Quartier-la-Tente M. 2008. - Ammonites sinémuriennes du Haut-Atlas marocain. Mémoires de Géologie de Lausanne 47: 1-99.
Haug E. 1911. - Traité de Géologie. II. Les périodes géologiques. Librairie Armand Colin, Paris, fasc. 2: 929-1396.

HowARTH M. K. 2002. — The Lower Lias of Robin Hood's Bay, Yorkshire, and the work of Leslie Bairstow. Bulletins of the Natural History Museum 58: 81-152. https://doi.org/10.1017/s0968046202000037

HowarTH M. K. 2013. - Treatise on Line, number 57, part L, Revised, Volume 3B, Chapter 4: Psiloceratidae, Eoderoceratoidea, Hildoceratoidea. Palaeontological Institute, University of Kansas, Lawrence, Kansas, 139 p. https://doi.org/10.17161/to.v0i0.4441

Hug O. 1899. - Beiträge zur Kenntnis der Lias und DoggerAmmoniten aus der Zone des Freiburger Alpen. Teil 2: Die Unter- und Mittellias Ammoniten fauna von Blumensteinallmend and Langeneckgrat am Stockhorn. Schweitzerische Paäontologische Abhandlungen 26: 1-39.

JohanNSON G. G., SMith P. L. \& Gordey S. P. 1997. — Early Jurassic evolution of the northern Stikinian arc: évidence from the Laberge Group, northwestern British Columbia. Canadian Journal of Earth Sciences 34: 1030-1057. https://doi.org/10.1139/e17-085

Kamoun F., Peybernès B. \& Fauré PH. 1999. - Évolution paléogéographique de la Tunisie saharienne et atlasique au cours du Jurassique. Comptes Rendus de l'Académie des Sciences de Paris 328 (8): 547-552. https://doi.org/10.1016/S1251-8050(99)80137-2

Lachkar N., Dommergues J.-L., Meister C., IZARd A. \& LANG J. 1998. - Les ammonites du Sinémurien supérieur du Jebel Bou-Hamid (Haut-Atlas central, Rich, Maroc). Approches paléontologiques et biostratigraphiques. Geobios 31: 587-619. https:// doi.org/10.1016/S0016-6995(98)80046-6

MeISTER C. \& BÖHM F. 1993. - Austroalpine Liassic Ammonites from the Adnet Formation (Northern Calcareous Alps). Jahrbuch der Geologischen Bundesanstalt 136 (1): 163-211.

Meister C. \& Friebe J. G. 2003. — Austroalpine Liassic Ammonites from Vorarlberg (Austria, Northern Calcareous Alps). Beiträge zür Paläontologie 28: 9-99.

Meister C., Blau J., Dommergues J.-L., Schlatter R. \& SchmidtEFFING R. 2009. - The Upper Sinemurian Ammonite succession in the Sierra Madre Oriental (Mexico). Volumina Jurassica 7: 31-36.

Meister C., Schirolli P. \& Dommergues J.-L. 2017. — Early Jurassic (Sinemurian to basal Toarcian) Ammonites of the Brescian Prealps (Southern Alps, Italy). Rivista Italiana di Paleontologie e Stratigrafia 123 (1): 79-148

Meneghini G. 1853. - Nuovi fossili toscani, in SAVI P. \& MenEGHINI G. (eds), Considerazioni sulla Geologia stratigraphica della Toscana. Annali dell'Università Toscana 3: 55-75. https:// www.biodiversitylibrary.org/page/9285583

MenEGHINI G. 1884. — Ellipsatiniae del Gargano e di Gebel Erssas in Tunisia. Atti della Societa toscana di Scienze Naturale, P.V. 4: 106.

MENEGHINI J. 1867-1881. — Monographie des fossiles du Calcaire rouge ammonitique (Lias supérieur) de Lombardie et de l'Apennin de l'Italie centrale, in STOPPANi A. (ed.), Paléontologie lombarde. Vol. 4. Stoppani ed., Milan, $242 \mathrm{p}$.

Mouterde R. 1965. - Sur quelques ammonites du Lias du Rif. Remarques paléontologiques. Notes et Mémoires du Service géologique du Maroc 184: 227-243.

Mouterde R., Corna M., Olivier Ph. \& Mourier T. 1986. Ammonites d'affinités mésogéennes dans le Lias inférieur des blocs prédorsaliens du Rif (Maroc), in PICG UNESCO, n 183 Marrakech 1985. Revue de la Faculté des Sciences de Marrakech, Section Sciences de la Terre, numéro spécial 2: 349-367

OrbignY A. D' 1842-1851. - Paléontologie française; Terrains jurassiques. 1. Céphalopodes. Masson, 642 p. https://doi.org/10.5962/ bhl.title. 50510

PAGE K. N. 2003. - The Lower Jurassic of Europe: its subdivision and correlation. Geological Survey of Denmark and Greenland Bulletin 1: 23-59. https://doi.org/10.34194/geusb.v1.4646

PALFY J. 1991. - Uppermost Hettangian to Lowermost Pliensbachian (Lower Jurassic) Biostratigraphy and Ammonoid Fauna of the Queen Charlotte Islands, British Columbia. Thesis British Columbia University, $243 \mathrm{p}$. 
PALFY J. \& SCHMidT K. L. 1994. - Biostratigraphic and facies studies of the Telkwa Formation (Lower Jurassic), Smithers map area, British Columbia. Geological Survey of Canada, Current Research 1994-E: 29-38.

PARONA C. F. 1896. - Contribuzione alla conoscenza delle Ammoniti liasiche di Lombardia. Parte I: Ammoniti del Lias inferiore. Mémoires de la Société paléontologique Suisse 23: 1-45.

Pervinquière L. 1903. - Etude géologique de la Tunisie centrale. Thèse de Doctorat de l'Université de Paris et Direction générale des Travaux publics, Tunis, $359 \mathrm{p}$.

PeYBernès B. 1992. - The Jurassic of Tunisia: attempt of reconstruction of the South-Neotethyan margin during and after the rifting phase; importance of ante-Bathonian paleostructures, in SAlEM M. J. \& BusReWIL M. T. (eds), Geology of Lybia. Elsevier, Tripoli: $1679-1766$

Peybernès B., Kamoun F., Durand-Delga M., Thierry J., Faure Ph., Dommergues J.-L., Vila J.-M., Cugny P. \& Ben Youssef M. 1996. — Le Jurassique et le Crétacé basal de la Tunisie atlasique nord-occidentale: essai de corrélations avec les formations de la Dorsale tunisienne et de la «ride» Amar-Djédeida. Comptes Rendus de l'Académie de Sciences de Paris 323, IIa: 153-162.

PINI S. \& SALAJ J. 1969. - Découverte du Jurassique à l'Ouest de Medjez-El-Bab. Notes du Service géologique de Tunisie 31: 25-35.

PozZi R. 1960. — La fauna liassica dell'Alta Valtellina (Alpi retiche). Rivista Italiana di Paleontologia e stratigrafia 66 (4): 445-499.

QuenstedT F. A. 1846-1849. - Petrefactenkunde Deutschlands. I: Die Cephalopoden. Fuess, Tübingen, 580 p., 36 pls.

RAKÚs M. 1973. — Le Jurassique du Djebel Ressas (Tunisie septentrionale). Livre jubilaire Marcel Solignac. Annales des Mines et de la Géologie 26: 137-147.

RAKÚS M. 1994. - Les ammonites lotharingiennes du Jbel BouHamid (Haut-Atlas de Rich, Maroc). Palaeopelagos, Special Publication 1: 299-316.

RAKÚS M. 1999. - Liassic ammonites from Hierlatz, Austria. Abhandlungen der geologischen Bundesanstalt 56 (2): 343-377.

RAKÚs M. \& BIELY A. 1970. - Stratigraphie du Lias dans la Dorsale tunisienne. Notes du Service géologique de Tunisie 32:45-64.

RAKÚs M. \& GUEX J. 2002. — Les ammonites du Jurassique inférieur et moyen de la Dorsale tunisienne. Mémoires de Géologie 39: $1-217$.

REYNÈS P. 1879. - Monographie des Ammonites. 1erpartie. Lias. Atlas. Baillères, Marseille et Paris, 72 p., 50 pls.

ROUVIER H. 1977. - Géologie de l'extrême-nord tunisien: tectoniques et paléogéographies superposées à l'extrémité orientale de la Chaîne maghrébine. Thèse Doctorat d'état, Université Pierre et Marie Curie, 2 Vols, 703 p. et 195 p.

SACCHI-Vialli G. \& CANTALUPPI G. 1961. — Revisione della fauna di Saltrio. Atti dell'Istituto geologico della università di Pavia 12: 5-49.

SCHAFHÄULT K. E. V. 1847. — Die Stellung des Bayerischen Voralpen im geologisches system. Neues Jahrbuch für Mineralogie, Geognosie Geologie und Petrefaktenkunde: 803-812.
SCHAFHÄUlt K. E. V. 1851. - Geognostische Untersuchungen des südbayerischen Alpengebirges. Vol. 33. Literarisch-artitische Anstalt, München, 206 p.

SCHLEgelmilch R. 1992. - Die Ammoniten des Süddeutschen Lias. Ein Bestimmungsbuch für Fossiliensammler und Geologen. 2. Auflage. G. Fischer Verlag, Stuttgart, Jena, New-York, 241 p. SEKATNI-Aich N. 2010. - Le Jurassique de Jebel Bou Kornine d'HammamLif. Stratigraphie, dynamique sédimentaire et évolution structurale. Thèse de Doctorat de l'Université de Tunis el Manar, 291 p.

SeKatni N., Fauré Ph., Alouani R. \& Zargouni F. 2008. — Le passage Lias-Dogger de la Dorsale de la Tunisie septentrionale. Nouveaux apports biostratigraphiques. Âge toarcien de la distension téthysienne. Comptes Rendus Palevol 7: 153-160. https://doi. org/10.1016/j.crpv.2008.03.001

Sekatni N., Fauré Ph., Arfaoui S. Alméras Y. \& Zargouni F. 2013. - La Formation Bent Saïdane dans le Jebel Bou Kornine d'Hammam-Lif (Bajocien supérieur à Callovien inférieur, Tunisie septentrionale). Nouvelles datations de ses deux niveaux conglomératiques. Interprétations paléogéographique et géodynamique. Bulletin de la Société d'Histoire naturelle de Toulouse 149: 179-190.

SMITH P.-L. 1981. - Biostratigraphy and Ammonoid Fauna of Lower Jurassic (Sinemurian, Pliensbachian an Lowest Toarcien) of Eastern Oregon and Western Nevada. Thesis Hamilton (Ontario), XXIV + $368 \mathrm{p}$

SOLIGNAC M. 1927. - Étude géologique de la Tunisie septentrionale. Thèse de la Faculté des Sciences de Lyon, Imprimerie Barlier \& Cie, Tunis, 756 p.

Soussi M. 2002. — Le Jurassique de la Tunisie atlasique. Stratigraphie, dynamique sédimentaire, paléogéographie et intérêt pétrolier. Documents des Laboratoires de Géologie de Lyon 157: 1-363.

SuAREZ-VeGA L. C. 1974. - Estratigrafia del Jurassico en Asturias. Cuadernos de Geologia iberica 1 (3): 1-304.

Thévenin A. 1907. - Types du Prodrome de paléontologie stratigraphique universelle de d'Orbigny. Annales de Paléontologie 2 (2): 89-96

THIERRY J. et al. (40 co-authors) 2000. — Late Sinemurian (193-191 Ma), in Dercourt J., Gaetani M., VRIelinck B., Barrier E., Biju-Duval B., Bruner M. F., Cadet J. P., Crasquin S. \& SanDulescu M. (eds), Atlas Peri-Tethys, Paleogeographic Maps. Explanatory Notes. CCGM-CGMV, Paris, map ${ }^{\circ} 7$.

TopChishVili M., Lominadze T., Tséreteli I., TODRIA V. \& NADAREISHVILI 2006. - Stratigraphy of the Jurassic deposits of Georgia. Georgian Academy of Sciences 122: 1-453.

Trueman A. E. \& Williams D. S. 1925. - Studies in the Ammonites of the Family Echioceratidae. Transactions of the Royal Society of Edimburg 53 (3): 699-739. https://doi.org/10.1017/ S0080456800027551

Wilmsen M., Blau J., Meister C., Mehdi M. \& Neuweiler F. 2002. - Early Jurassic (Sinemurian to Toarcian) ammonites from the central Hight Atlas (Morocco) between Errachidia and Rich. Revue de Paléobiologie 21 (1): 149-175. 